\title{
How the incorporation of Pluronic block copolymers modulates the response of lipid membranes to mechanical stress
}

DOI:

10.1021/acs.langmuir.7b02244

\section{Document Version}

Accepted author manuscript

Link to publication record in Manchester Research Explorer

Citation for published version (APA):

Zaki, A. M., \& Carbone, P. (2017). How the incorporation of Pluronic block copolymers modulates the response of lipid membranes to mechanical stress. Langmuir. https://doi.org/10.1021/acs.langmuir.7b02244

\section{Published in:}

Langmuir

\section{Citing this paper}

Please note that where the full-text provided on Manchester Research Explorer is the Author Accepted Manuscript or Proof version this may differ from the final Published version. If citing, it is advised that you check and use the publisher's definitive version.

\section{General rights}

Copyright and moral rights for the publications made accessible in the Research Explorer are retained by the authors and/or other copyright owners and it is a condition of accessing publications that users recognise and abide by the legal requirements associated with these rights.

\section{Takedown policy}

If you believe that this document breaches copyright please refer to the University of Manchester's Takedown Procedures [http://man.ac.uk/04Y6Bo] or contact uml.scholarlycommunications@manchester.ac.uk providing relevant details, so we can investigate your claim.

\section{OPEN ACCESS}




\section{Langmuir}

This document is confidential and is proprietary to the American Chemical Society and its authors. Do not copy or disclose without written permission. If you have received this item in error, notify the sender and delete all copies.

\section{How the incorporation of Pluronic block copolymers modulates the response of lipid membranes to mechanical stress}

\begin{tabular}{|r|l|}
\hline Journal: & Langmuir \\
\hline Manuscript ID & la-2017-02244g.R1 \\
\hline Manuscript Type: & Article \\
\hline Date Submitted by the Author: & $08-S e p-2017$ \\
\hline Complete List of Authors: & $\begin{array}{l}\text { Zaki, Afroditi; The University of Manchester, School of Chemical } \\
\text { Engineering and Analytical Science } \\
\text { Carbone, Paola; The University of Manchester, School of Chemical } \\
\text { Engineering and Analytical Science }\end{array}$ \\
\hline & \\
\hline
\end{tabular}

SCHOLARONE

Manuscripts 


\title{
How the incorporation of Pluronic ${ }^{\circledR}$ block copolymers modulates the response of lipid membranes to mechanical stress
}

\author{
Afroditi Maria Zaki* and Paola Carbone* \\ School of Chemical Engineering and Analytical Science, The University of Manchester, \\ Oxford Road, Manchester, M13 9PL, United Kingdom \\ E-mail: afroditimaria.zaki@manchester.ac.uk; paola.carbone@manchester.ac.uk
}

\begin{abstract}
We employ atomistic Molecular Dynamics simulations to investigate the effect that the incorporation of the nonionic amphiphilic copolymer known as Pluronic ${ }^{\circledR}$ L64 has on the mechanical stability of a DPPC membrane. The simulations reveal that the incorporation of the polymer chains leads to membranes that can sustain increasing mechanical stresses. Analysis of mechanical, structural and dynamic properties of the membrane shows that the polymer chains interact strongly with the lipids in the vicinity, restraining their mobility and imparting better mechanical stability to the membrane. The hybrid membranes under tension remain thicker, more ordered and stiffer in comparison to their lipid analogues. Trans-bilayer lipid movements (flip-flop) are observed and appear to be triggered by the presence of the polymer chains. A careful analysis of the pore formation under high tensions reveals two distinctive mechanisms that depend on the distribution of the hydrophilic polymer blocks in the bilayer. Finally, the rate of growth of the formed membrane defects is slowed down in the presence of polymers. These findings show that Pluronic ${ }^{\circledR}$ block copolymers could be exploited
\end{abstract}


for the formation of optimized hybrid nanodevices with controlled elastic and dynamic properties.

\section{Introduction}

The mechanism by which polymers modify and interact with lipid bilayers is a crucial step for the development of new polymer-based drug nano-carriers. ${ }^{1-4}$ Such nanocarriers can be developed entirely from polymers (polymersomes, micelles, dendrimers), ${ }^{5}$ or, to minimize the side effects, be self-assembled from a mixture of polymers and lipids (hybrid particles). The latter type of vesicles should have the advantage of retaining the good mechanical properties typical of the polymer vesicles and of displaying milder adverse effects. ${ }^{5,6}$ For example it has been shown that grafting lipid vesicles with Polyethylene glycol (PEG) increases the vesicles' stability during blood circulation ${ }^{7,8}$ and that polyelectrolyte-coated DOPC vesicles possess improved mechanical stability under $\mathrm{pH}$ shock. ${ }^{9,10}$ In particular, the incorporation of amphiphilic block copolymers in phospholipid vesicles have shown to enhance not only the vesicles' mechanical stability but also the loading rates of drugs and the drug cellular uptake. ${ }^{11,12}$ Experimental data showed that these hybrid vesicles have substantially increased shelf stability, resistance against rupture and fusion, and are characterized by decreased water permeability. ${ }^{8,13}$ In addition, recent in-vivo studies on mice showed that amphiphilic block copolymer-lipid vesicles loaded with an anticancer drug cocktail cause reduced organ toxicity and higher drug load release at the target cells. ${ }^{14}$ While the data clearly indicate that the hybrid polymer-lipid vesicles possess improved mechanical properties, it is less clear what molecular mechanism drives such changes. The polymer molecular weight, its microstructure and, more complicated to rationalize, the procedure followed for the self-assembly seem to be all variables affecting the final stability of the vesicles. ${ }^{6,11}$ Specifically, for amphiphilic copolymers, the hydrophobic/hydrophilic ratio and the molar mass of the polymers seem to be the main factors affecting both the cellular uptake and the change in the membrane 
properties. ${ }^{15}$ Due to the difficulty in gaining an atomistically resolved picture of the vesicles' structure, molecular simulations have been extensively used to investigate the effect that polymers, surfactants or small molecules have on the structure of lipid bilayers. ${ }^{16-22}$

Specifically, Srinivas et al. ${ }^{23}$ have firstly shown, using coarse-grained simulations, that diblock copolymers of polyethylene glycol (PEG-PEE) translocate within preformed lipid bilayers that consequently increase their thickness. The simulations showed that the hydrophilic block tends to reside at the water/bilayer interface while the hydrophobic block moves inside the bilayer tail region. Despite the numerous computational studies on polymer/lipid membranes, most of them focus on structural and dynamic properties of stress-free systems and there is a lack in the investigation of the membranes' mechanical properties. One of the few such studies, is the work by Tomasini et al. ${ }^{24}$ who, using a mesoscopic model, showed that the (PEG-PEE) copolymer modifies the bilayer mechanical properties and highlighted how the hydrophilic-lipophilic balance and the polymer chain molecular weight can either enhance or reduce the vesicles mechanical stability.

Among the various amphiphilic block copolymers that can be employed to fabricate these hybrid vesicles, Pluronic ${ }^{\circledR}$, a series of triblock copolymers of ethylene oxide (EO) and propylene oxide (PO) arranged along the chain as a EO-PO-EO sequence, look particularly interesting. Due to their amphiphilic nature, in aqueous environments they form micelles, ${ }^{25}$ which are suitable for the encapsulation of drugs, proteins and genes and can therefore be used as nanocarriers. Their properties can be controlled by tuning the hydrophilic-lipophilic balance and their molecular weight. Being already FDA approved, they are currently widely used in the cosmetic and pharmaceutical industry, ${ }^{15}$ however they demonstrate increased levels of toxicity if used in high dosage. On the other hand, Pluronic ${ }^{\circledR}$ coated nanoparticles developed for medical imaging purposes were characterized as highly biocompatible and relatively non-toxic, ${ }^{15}$ opening the possibility for Pluronic ${ }^{\circledR}$ copolymers to be employed in hybrid systems for nanomedical applications. Experimental data ${ }^{26,27}$ and molecular simulations ${ }^{17,21,28}$ on stress-free lipid bilayers containing different types of Pluronic ${ }^{\circledR}$ copolymers 
have already showed that the incorporation of the chains within the bilayer alters the membrane thickness, the lipid diffusivity and packing properties and the bilayer permeability. The data have also suggested that in some cases the amphiphilic copolymers are able to heal a damaged membrane by stabilizing its structure, but in other cases, that the presence of the copolymers disturbs the lipid packing and enhances membrane permeability. ${ }^{29}$

The aim of this work is to elucidate the impact that the incorporation of Pluronic ${ }^{\circledR}$ chains has on various properties of phospholipid bilayers when the membrane is subjected to mechanical stresses. This work focuses specifically on a Pluronic ${ }^{\circledR}$ labeled as L64 (consisting of a $40 \%$ EO content and a PO molecular weight of $1800 \mathrm{~g} / \mathrm{mol}^{30}$ ) (Figure 1a) which is one of the best experimentally characterized among the copolymer series ${ }^{31-37}$ and shows spontaneous adsorption into phospholipid bilayers. ${ }^{38}$

The well characterized 1,2-dipalmitoyl-sn-glycero-3-phosphocholine (DPPC) lipid (found in abundance in mammalian cell membranes as one of their main constituents) was selected for the construction of the model lipid bilayer ${ }^{39,40}$ (Figure 1b). It is a zwitterionic phospholipid and has been used -among others- for the development of various types of hybrid nanoparticles, ${ }^{11}$ including binary polymer/lipid mixtures, ${ }^{12}$ core-shell type polymerlipid nanoparticles ${ }^{6,11}$ and polymer-caged liposomes. ${ }^{41}$

(a) $\mathbf{L 6 4}$

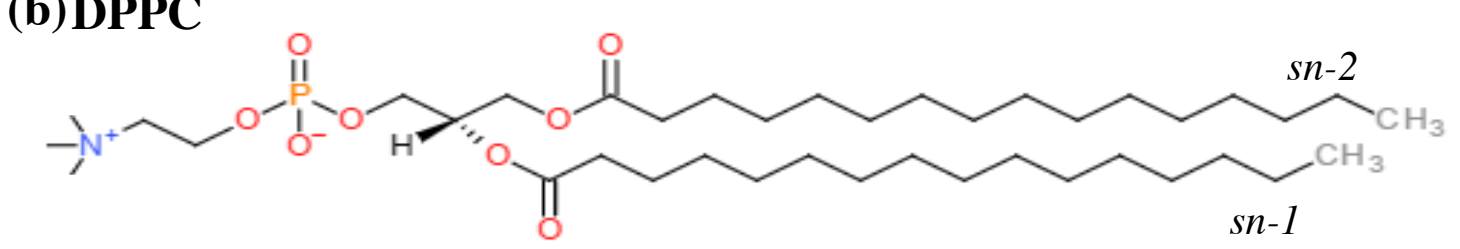

Figure 1: (a) Molecular structure of the L64 Pluronic ${ }^{\circledR}$. The blocks in brackets highlight the ethylene oxide (EO) (green) and the propylene oxide (PO) (red) segments. (b) Molecular structure of the DPPC lipid. 


\section{Methodology}

\section{Systems}

Lipid Bilayers. The Berger et al. ${ }^{42}$ force field and the single point charge SPC model ${ }^{43}$ were used to model the lipid and the water molecules, respectively. The lipid bilayer was built starting from a previously equilibrated DPPC lipid bilayer consisting of 128 lipids and 3655 water molecules, downloaded from http://wcm.ucalgary.ca/tieleman/downloads/dppc.itp. The water molecules were then removed and the box was duplicated in the $x$ - and $y$ - dimensions to generate a bilayer of 512 lipids which was then placed in the center of a box of dimensions $13 \times 13 \times 10 \mathrm{~nm}^{3}$. Approximately 33000 water molecules were added and the system was then energy minimized following the steepest descent method and equilibrated in an NVT and subsequently, in an NPT ensemble.

Polymer-Lipid Bilayers. Hybrid polymer-lipid (HPL) systems were obtained by adding L64 polymer chains to the water phase of the pure lipid membrane system and allowing the polymers to insert into the preformed bilayer. The L64 polymer was modeled using the unmodified GROMOS force field with partial charges obtained from DFT calculations. For the method followed for the development and validation of the force field parameters, we refer the reader to the original work. ${ }^{17}$ For convenience, the set of charges is reported in the supporting information (Table S1).

In order to elucidate the effect of polymer concentration, either one or twelve L64 chains were added resulting in polymer mass fractions in the polymer-lipid mixture of approximately $1.2 \mathrm{wt} \%$ and $12.7 \mathrm{wt} \%$ (in DPPC/L64 molar ratios this translates to 82:1 and 7:1, respectively). In the text, the hybrid systems will be labeled as LowHPL (DPPC/L64 82:1) and HighHPL (DPPC/L64 7:1) for brevity. The twelve L64 chains were initially randomly distributed in the entire $x y$-plane covering most of the surface of one of the leaflets and acting as coating, mimicking the preparation of polymer-coated liposomes. ${ }^{2}$ Finally, the space was again filled with enough water molecules to fill a box of similar size as the pure lipid 
system. Energy minimization was carried out followed by an NVT and an initial NPT equilibration with the polymer chains being position restrained. This initial equilibration step was necessary to relax the water molecules around the polymer and the lipids and to ensure that the thermodynamic properties have equilibrated before allowing the polymers to move freely. The restraints were then removed and the systems were subjected to an additional 100 ns NPT run within which the polymer chains translocate and remain incorporated in the bilayer. The final configurations were used as the starting points for the production runs where the membranes were expanded as will be described below.

\section{Simulations under mechanical stress}

In order to assess the resistance of the membranes under tension, both the $\mathrm{NP}_{N} \mathrm{AT}$ (constant surface area) and NPT (isothermal-isobaric) thermodynamic ensembles were preliminary tested on the DPPC bilayers. Specifically for the NPT ensemble, two loading rates were applied and the results (i.e., area per lipid and surface tension) obtained from the three different procedures were compared. For the high loading rate NPT simulations (NPT-HR), lateral pressures $\left(P_{L}\right)$ of increasing magnitude ranging from -10 bar to $P_{L C}\left(P_{L C}\right.$ : critical rupture lateral pressure) were applied on the equilibrated tensionless membrane. On the contrary, for the low loading rate simulations (NPT-LR), the lateral pressure was applied to the final configuration of each previously equilibrated NPT simulation. The latter procedure of gradually increasing the pressure allowed the system to adjust to the expansion and produced comparable area-per-lipid and surface tension data with the $\mathrm{NP}_{N} \mathrm{AT}$ ensemble simulations (Figure S1) and therefore was selected as the method to expand the HPL systems. The pressure coupling was semi-isotropic, with the pressure applied in the direction normal to the membrane $\left(P_{N}\right)$ (set at 1 bar) being coupled separately from the lateral pressure $\left(P_{L}\right)$. The temperature was set at $323.15 \mathrm{~K}$, approximately $8 \mathrm{~K}$ above the gel-to-liquid phase transition temperature of unsupported DPPC. ${ }^{44}$ The temperature and pressure were controlled with the Nose-Hoover thermostat ${ }^{45-47}$ and the Parrinello-Rahman barostat ${ }^{48,49}$ 
with time constants of $0.5 \mathrm{ps}$ and $5 \mathrm{ps}$, respectively. Periodic boundary conditions were applied in all three dimensions. The bonds were constrained using the LINCS algorithm. ${ }^{50}$ The Particle-Mesh Ewald summation ${ }^{51}$ was adopted for the treatment of the long range electrostatic interactions, as it produces the most reliable results for systems with charges. A cubic interpolation was chosen with a grid spacing of $0.16 \mathrm{~nm}$. The Lennard-Jones interactions and the short range Coulomb interactions were cut-off at $1 \mathrm{~nm}$. The time step was $2 \mathrm{fs}$ and the leap-frog algorithm was selected for the integration of the equations of motion. The total length of each production run was $500 \mathrm{~ns}$, of which the final 50-200 ns were used for the calculations, depending on the type of analysis. The MD simulations were performed using the GROMACS software package (version 4.6.5). ${ }^{52}$ The VMD software (version 1.9) ${ }^{53}$ was used for the visualisation of the systems and the MDAnalysis package ${ }^{54,55}$ was employed for the development of home-made analysis tools.

\section{Results and Discussion}

\section{Effect of the L64 chains on the elastic properties of the membrane}

Within few nanoseconds after removing the position restraints, the polymer chains started to interact with the headgroups of the phospholipids and translocated into the bilayer where they remained adsorbed for the total length of the simulation (Figures $2 \mathrm{~b}$ and $2 \mathrm{c}$ ). This observation was not surprising as the poloxamers are surface-active polymers and rapidly adsorb onto oil-water interfaces ${ }^{56-60}$ and one of their main applications is as stabilizers for oil/water emulsions. ${ }^{38,57}$ Most of the polymer chains remained localized in the upper leaflet and the hydrophobic nature of the PO segment of the polymers allowed, within the simulation time, only few of the chains to penetrate into the hydrophobic center of the bilayer and to move to the opposite leaflet (Figures 2c and 2f). The PEO block on the other hand, mostly lied close to the lipid/water interface, interacting closely with the hydrophilic heads of the lipids. The spatial distribution of the PEO blocks could depend on the polymer 
(a)

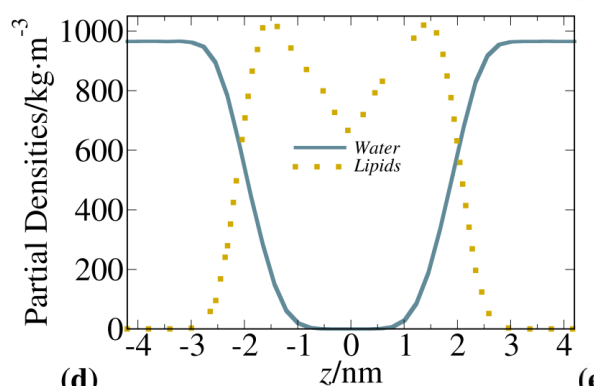

(d)

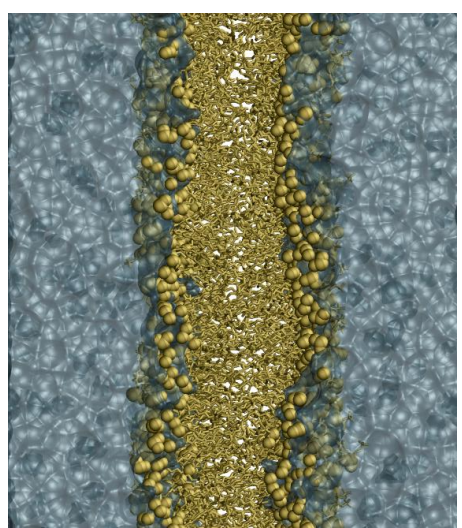

(b)
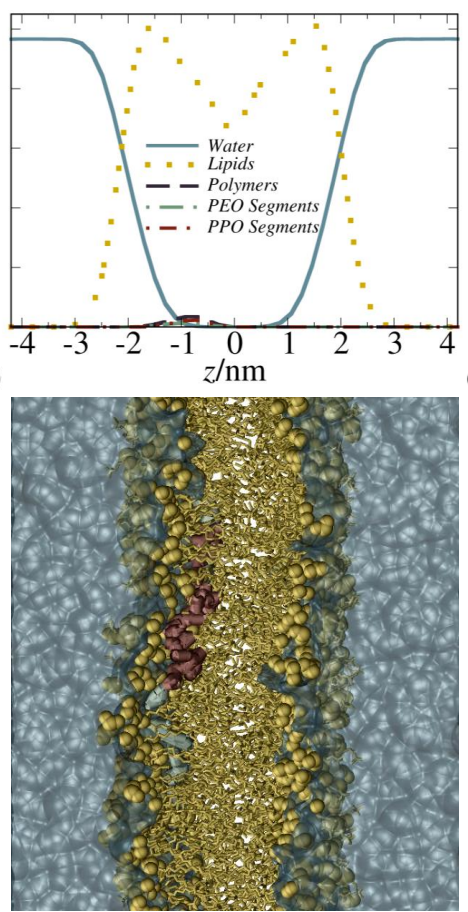

(c)

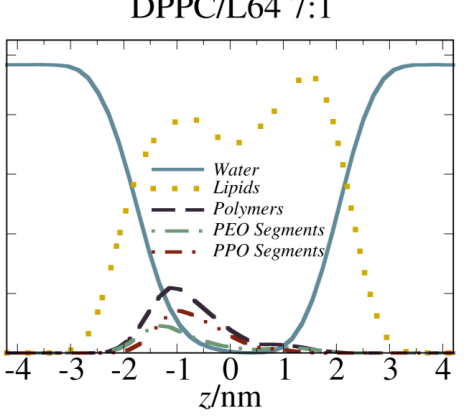

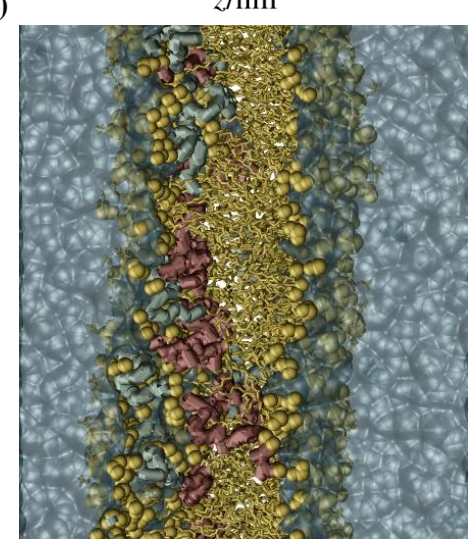

Figure 2: Upper Panel: Mass density profiles of all the system components in $P_{L}=1$ bar, averaged over the final $50 \mathrm{~ns}$ of each trajectory. Lower Panel: Representative snapshots of the membranes in water. (a),(d) DPPC; (b),(e) LowHPL; (c),(f) HighHPL. Color code of the snapshots: water in blue, lipids in yellow, polymer PEO blocks in green and polymer PPO blocks in red. 
content, as for similar mass fractions, the PEG blocks of amphiphilic copolymers have been found to behave similarly, whereas for higher polymer content, they were forced to extend in the water phase. ${ }^{23}$ Especially for the HighHPL system, the large difference in polymer concentration between the two leaflets induces a significant asymmetry in the membrane and has implications on its structural properties and fluidity, as it will be shown later (Figures 6, 7 and 8). The above equilibrated systems were then subjected to an increasingly high external lateral pressure as described in the Methodology section aiming to assess the mechanical properties of the membranes under tension. Experimentally, the mechanical strength of vesicles is investigated via the micropipette aspiration technique which probes the stressstrain curves and provides information on the membrane tension, the areal strain upon which lysis occurs and the area compressibility modulus $\left(K_{A}\right) \cdot{ }^{61}$ Similarly, the surface tension-areal strain curve can be obtained from the simulations carried out at different lateral pressures and is reported in Figure 3a. The surface tension $(\gamma)$ is calculated as:

$$
\gamma=L_{z}\left(P_{N}-P_{L}\right)
$$

where $L_{z}$ is the box length in the $z$-dimension, $P_{N}=P_{z z}$ and $P_{L}=\frac{1}{2}\left[P_{x x}+P_{y y}\right]$, with $P_{x x}, P_{y y}$ and $P_{z z}$ being the diagonal components of the pressure tensor. The areal strain $\left(\epsilon_{\mathrm{A}}\right)$ is defined as the relative increase in the membrane surface area under tension with respect to the surface area of a tensionless state:

$$
\epsilon_{A}=\frac{A_{i}}{A_{0}}-1
$$

where $A_{i}$ and $A_{0}$ are the bilayer surface areas in the systems under lateral pressure $P_{L i}$ and in the tensionless system, respectively.

As expected, the membranes responded to the applied pressure with an increase in their surface tension $(\gamma)$ as they tried to minimize the exposure of their hydrophobic constituents to water. All the membranes can withstand a 70-77\% expansion of their surface area prior 
the formation of a pore which leads to their rupture. For the polymer-free bilayer, the area per lipid at rupture is $\sim 1.1 \mathrm{~nm}^{2}$, which is in good agreement with previous studies of DPPC membranes under tension. ${ }^{16,62,63}$ The corresponding critical surface tension is approximately $20 \mathrm{mN} / \mathrm{m}$ lower than that found in previous computational work. ${ }^{16}$ This can be attributed to the larger size of the membrane patch simulated here, which is expected to lead to reduced bilayer cohesion and stability. ${ }^{16}$ The data show that low polymer content does not affect the response of the membrane to the applied pressure but that for high polymer content, the HighHPL membrane is characterized by higher surface tension compared to the other two systems, indicating stronger intermolecular attractive interactions at the interface. This increase in the surface tension upon addition of the copolymer is in disagreement with the results shown by Dissipative Particles Dynamics ${ }^{24}$ where however most of the hydrophilic part of the copolymer chains (PEO-PEE) resided at the outer part of the bilayer (e.g. towards the water phase). In the present case instead the PEO blocks are totally incorporated inside the membrane. The data also shows that, even though the rupture occurs at similar $\epsilon_{\mathrm{A}}$ in all cases, the HighHPL membrane can sustain an increased surface tension of $75.8 \mathrm{mN} / \mathrm{m}$ prior to failure compared to the pure lipid membrane that breaks at $71.8 \mathrm{mN} / \mathrm{m}$. Moreover, Figure $3 \mathrm{~b}$ shows that while in the case of the pure DPPC, the critical areal strain is reached for $P_{L c}=$ -110 bar, a higher lateral pressure of -130 bar is required to cause rupture in the HPL bilayers (Figure 3b). Here it is important to notice that the critical pressure $\left(P_{L c}\right)$ is a size-dependent property and that small deviations in the box dimensions affect its values which therefore should be interpreted with caution. Figure $3 \mathrm{~b}$ also demonstrates the resistance of the hybrid membranes to stretching when lateral pressure is imposed and how this resistance becomes more prominent at higher pressures. A significant increase in the lysis tension of HPL vesicles has also been found experimentally from Chen et al. ${ }^{12}$ who showed that the hybrid vesicles of phospholipids and PDMS-co-PEO copolymers are mechanically more stable than the pure lipid ones, as the critical areal strain and the critical rupture surface tension significantly increase when polymers are present and the vesicles can withstand higher expansion of their 
surface area before lysis. It is worth mentioning that the expansion the hybrid membrane can sustain before rupture in an experiment is much lower (no more than 8\%) than the critical strain calculated from simulations (exceeding 70\%). This discrepancy between experiments and simulations has been observed previously ${ }^{63}$ and is attributed to the dynamic nature of the pore formation process that depends on the loading rate. At the much higher loading rates applied in simulations, the critical rupture tension moves to higher values, as explained by the kinetic model proposed by Evans et al. ${ }^{64}$

The elasticity of a membrane can be assessed computationally and experimentally from the calculation of the compressibility modulus $\left(K_{A}\right)$ which is related to the resistance of a membrane to the expansion of its surface area when it is under mechanical stress. ${ }^{65}$ For small area expansion, it can be calculated via the slope of the stress-strain curve ${ }^{66}$ and for larger membrane expansion, it can be calculated via equation 3 :

$$
K_{A}=k_{B} T \frac{\langle A\rangle}{\left\langle\delta A^{2}\right\rangle}
$$

where $\langle A\rangle$ is the mean total area, $\left\langle\delta A^{2}\right\rangle$ is the mean square fluctuations of the area and $k_{B} T$ is the thermal energy. Membranes with high $K_{A}$, respond moderately to the applied stress showing a low areal strain for a given lateral tension and can be characterized as more cohesive. On the contrary, membranes that respond to the applied tension with high area fluctuations show increased elasticity. ${ }^{67,68}$ For the pure DPPC bilayer at zero surface tension, $K_{A}$ is found to be $247.7 \pm 30.0 \mathrm{dyn} / \mathrm{cm}$, which is in good agreement with the experimentally measured value of $231 \mathrm{dyn} / \mathrm{cm} .{ }^{39}$ Upon increasing tension, the compressibility modulus of both the polymer-free and hybrid membranes decreases (Figure 4), a trend also previously observed by Feller and Pastor ${ }^{66}$ for DPPC membranes. Looking at the impact of the polymers on $K_{A}$, it is clear that the LowHPL membrane behaves similarly to the pure lipid membrane, but when the polymer content is high, the membrane becomes stiffer (i.e., $K_{A}$ increases with respect to the pure lipid and the LowHPL systems), implying that higher energy would be required for its surface area to expand. This effect disappears when 
(a)

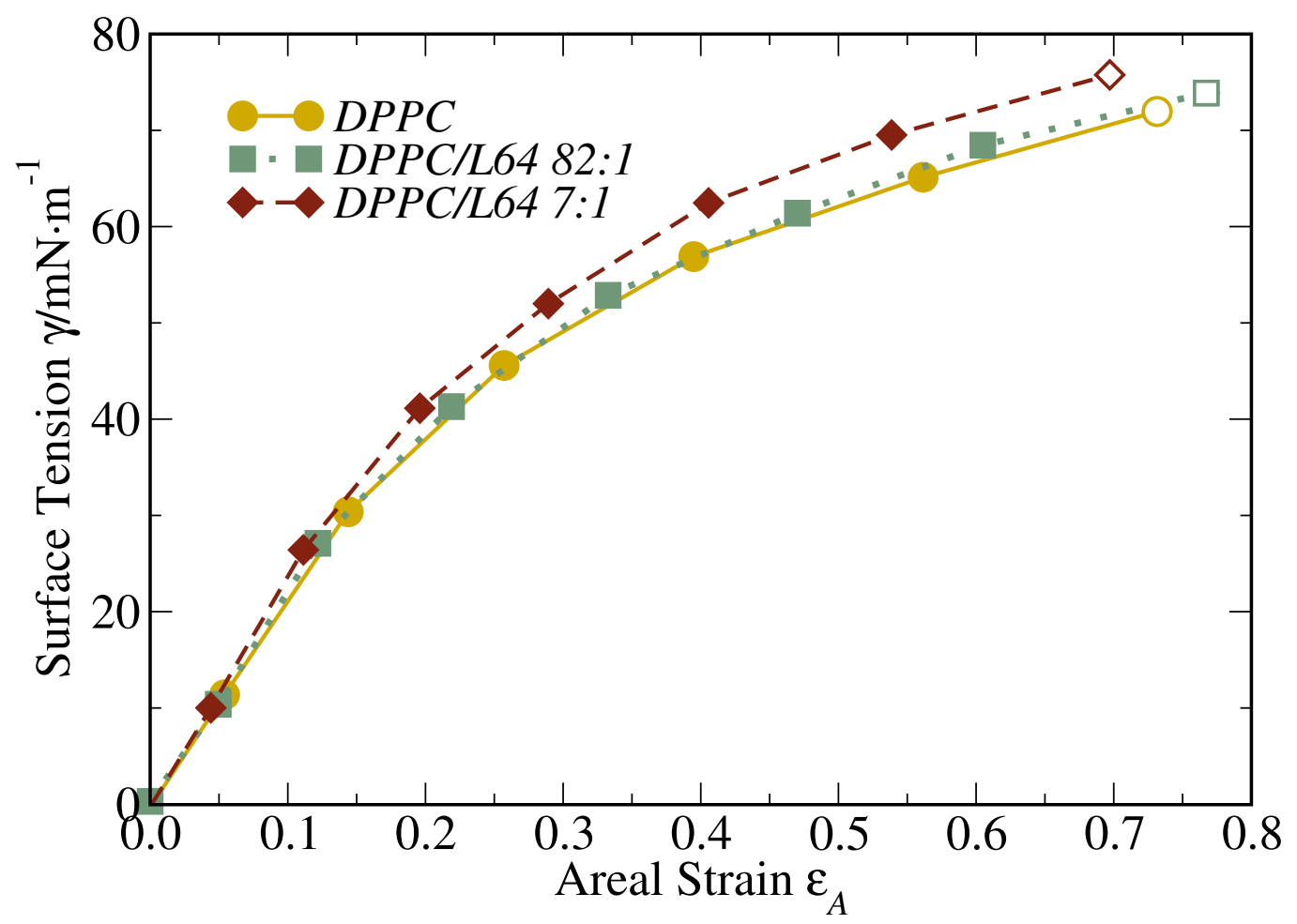

(b)

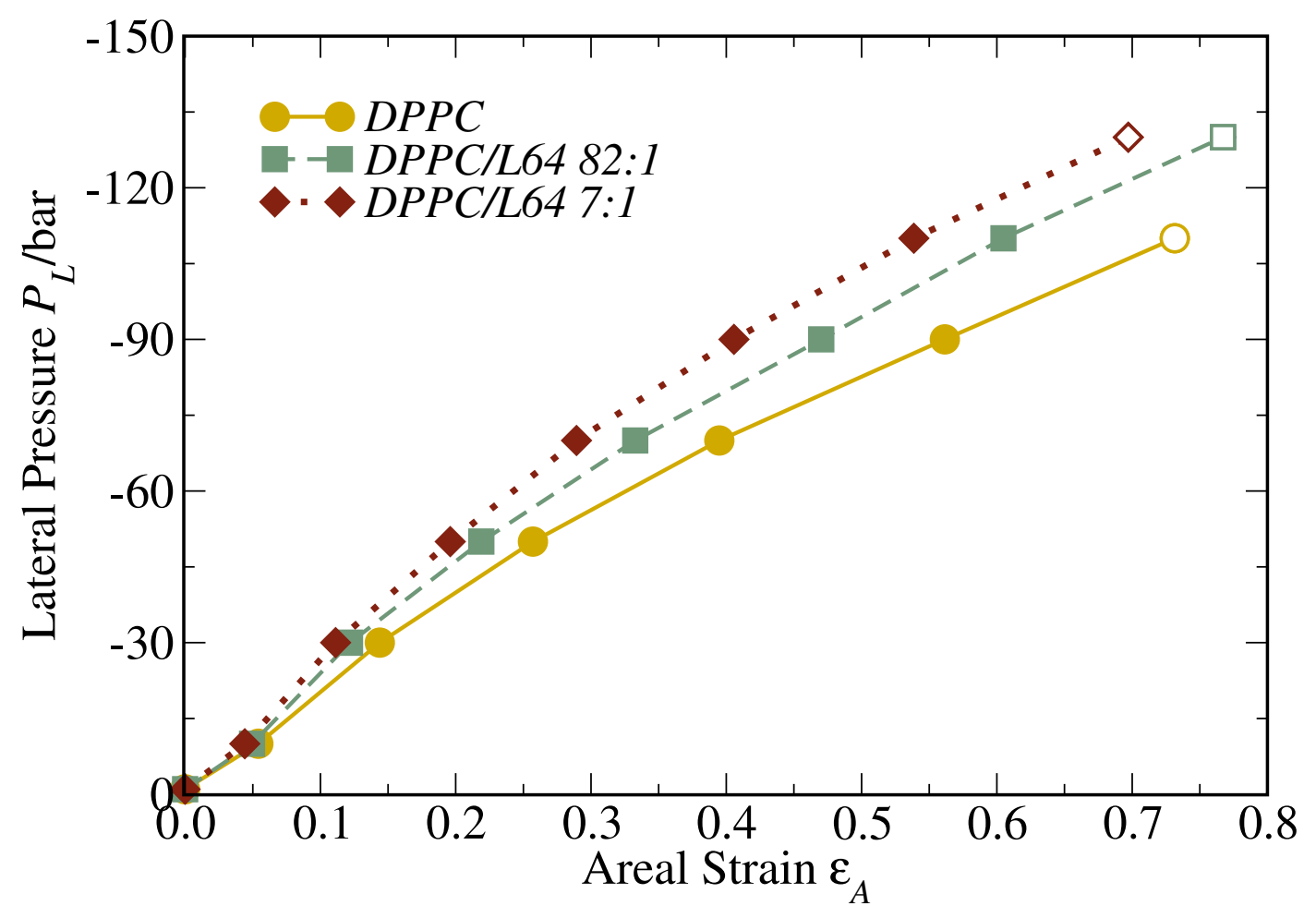

Figure 3: (a) Surface tension-areal strain curve and (b) Lateral pressure-areal strain curve for the two lipid/polymer ratios investigated here. The open symbols denote the rupture points. The standard errors of the mean are included, but are too small to be visible. 
$P_{L}$ becomes higher than -70 bar and above this pressure value all the membranes show a significant softening irrespective of their polymer content.

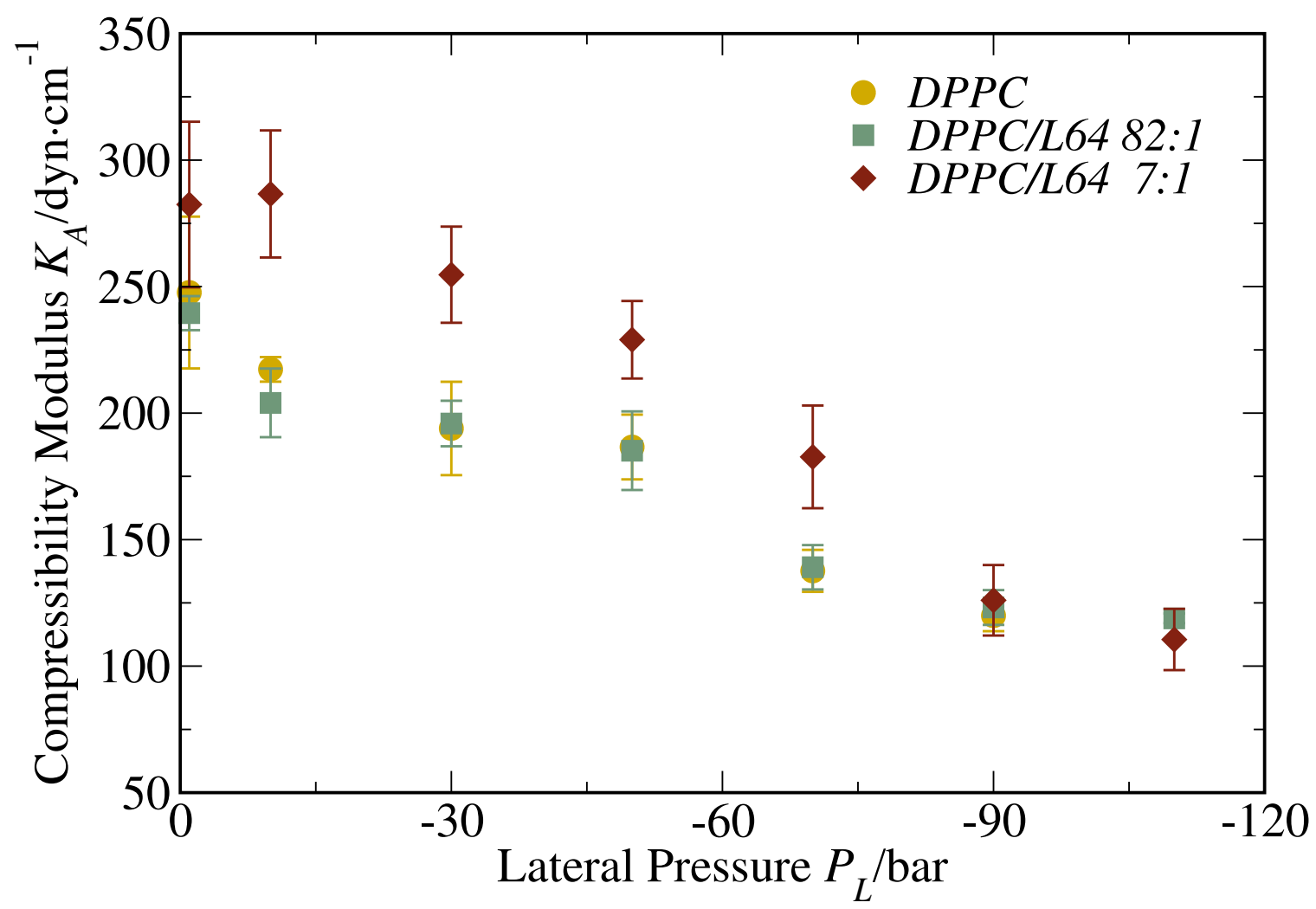

Figure 4: Area compressibility modulus $\left(K_{A}\right)$ of membranes with different lipid/polymer ratios versus the applied lateral pressures $\left(P_{L}\right)$. The error bars correspond to the standard errors of the mean, calculated from block averaging ${ }^{69}$ of four independent blocks of $100 \mathrm{~ns}$ each.

An initial understanding of the molecular mechanism behind the improved mechanical properties of the HPL membranes is obtained looking at the area $\left(A_{\text {occ }}\right)$ occupied by both the lipid and polymer molecules across the bilayer. To calculate $A_{o c c}$ we followed the method described in the work of Falck et al. ${ }^{68}$ and later also used by Nawaz et al., ${ }^{17}$ where the system is sliced across the $z$-axis (i.e., perpendicular to the membrane plane) and in each slice the atoms either belonging to the phospholipid molecules or to the polymer chain are mapped onto a two-dimensional grid. If a grid point lies within the Van der Waals radius of the atom, it is considered lipid-occupied or polymer-occupied, respectively. The total area profiles (Figure 5) reveal a thinning of the membrane as the expansion increases. For 
the polymer-free bilayer, the two leaflets present, as expected, a symmetric profile and the only effect that applying a lateral pressure has on the membrane is to increase -significantlyits surface area. This increased surface area forces the lipids to spread out resulting in an increased lipid-occupied area, $A_{\text {occ }}{ }^{1}$ For the system containing one polymer chain, a small difference in the area per lipid between the two leaflets is observed for low pressures, but at higher pressures this asymmetry vanishes. Comparing the profiles calculated for the HPL membranes to that for the lipid system, it can be seen how the presence of the polymer makes the bilayer more resistant to the expansion, as in the former the increase in the lipid-occupied area is more limited, especially as the pressure becomes high. Finally, the HighHPL system presents highly asymmetric profiles indicating that the applied tension has a much more pronounced effect on the structure of the polymer-free leaflet. The polymer chains occupy approximately $30 \mathrm{~nm}^{2}$ and are mainly located close to the headgroup region of one leaflet (the one from which they passively translocated inside the membrane), but some chains extend to the other leaflet and span the bilayer across the $z$-axis indicating that the polymer translocation is not inhibited by high polymer concentration.
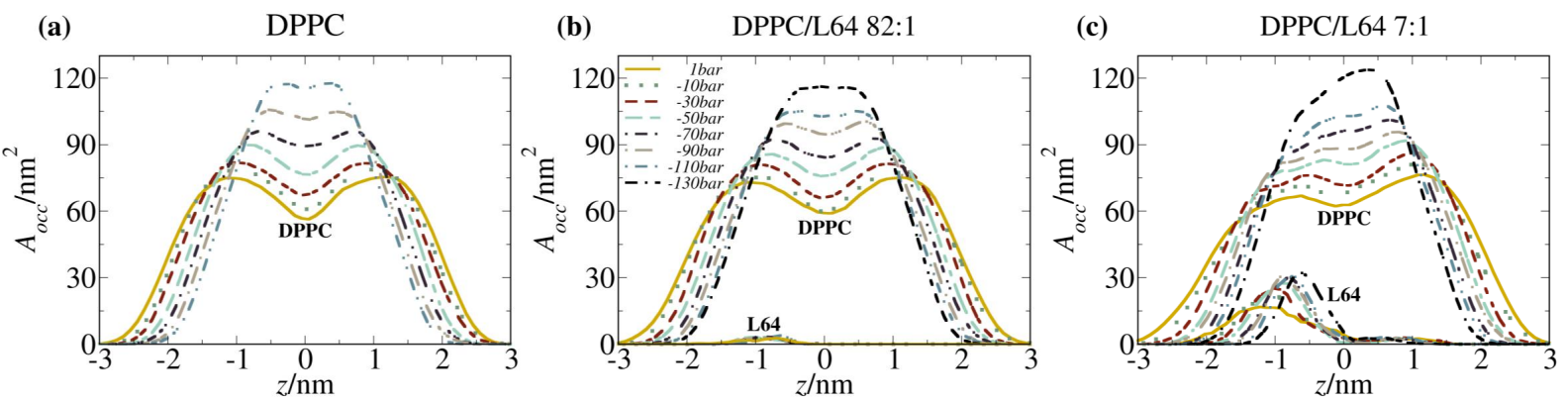

Figure 5: Lipid-occupied and polymer-occupied area profiles averaged over the final 10 frames of the trajectories, for all the studied lateral pressures $P_{L}$ below the critical rupture pressure $P_{L c}$. (a) Pure DPPC bilayer, (b) LowHPL bilayer and (c) HighHPL bilayer. Given that the models used for the lipids and the polymers are united-atom representations, the half of the force field $\sigma$ value was used as the Van der Waals radius. 


\section{Effect of the L64 chains on the structural properties of the membrane}

The ordering of the lipid acyl chains of the membrane was determined by calculating the carbon-deuterium order parameter $S_{C D}$ for the $s n$-1 acyl chains (see Figure 1) using equation 4:

$$
S_{z}=\frac{3}{2}\left\langle\cos ^{2} \theta_{z}\right\rangle-\frac{1}{2}
$$

where $\theta_{z}$ is the angle between the bilayer normal and the vector from $C_{n-1}$ to $C_{n+1}(C$ is the carbon atom). Since a united-atom model was used for the lipids, the C-D vector is replaced by a vector joining two successive carbon atoms on the same side of the acyl chain. The brackets indicate average over time and lipids. The order parameter $S_{z}$ is related to the deuterium order parameter as $S_{C D}=-\frac{1}{2} S_{z}$. The absolute value of $S_{C D}$ lies between 0 (isotropic orientation) and 0.5 (orientation along the bilayer normal). ${ }^{70}$

Irrespectively to the pressure applied and the presence of the polymer chains in the bilayer, the overall tendency is that the lipid ordering decreases with increasing distance from the lipid heads, approaching the value of zero at the terminal methyl groups, following the typical trend that has been previously observed both experimentally ${ }^{70}$ and computationally ${ }^{40,44}$ for tensionless membranes (Figure 6a). The orientation of the acyl chains is however clearly reduced when the membrane is expanded, a result that is rather expected, since the membrane is characterized by a higher free volume allowing the lipids to assume a large number of configurations and also a direct consequence of the membrane thinning (see above). However, a more interesting finding is that the polymers enhance the lipid tails' ordering, especially in the proximity of the headgroup region where the polymer chains are mostly located (see Figure 2). The effect is negligible for low lateral pressure, but becomes evident for $P_{L}$ exceeding the value of -30 bar. The polymer concentration also seems to play a role in the lipid tail packing as the values of the $S_{C D}$ are higher when more polymers are incorporated in the bilayer. Similar behavior is observed for the $s n$ - 2 fatty acid tails 
(a)

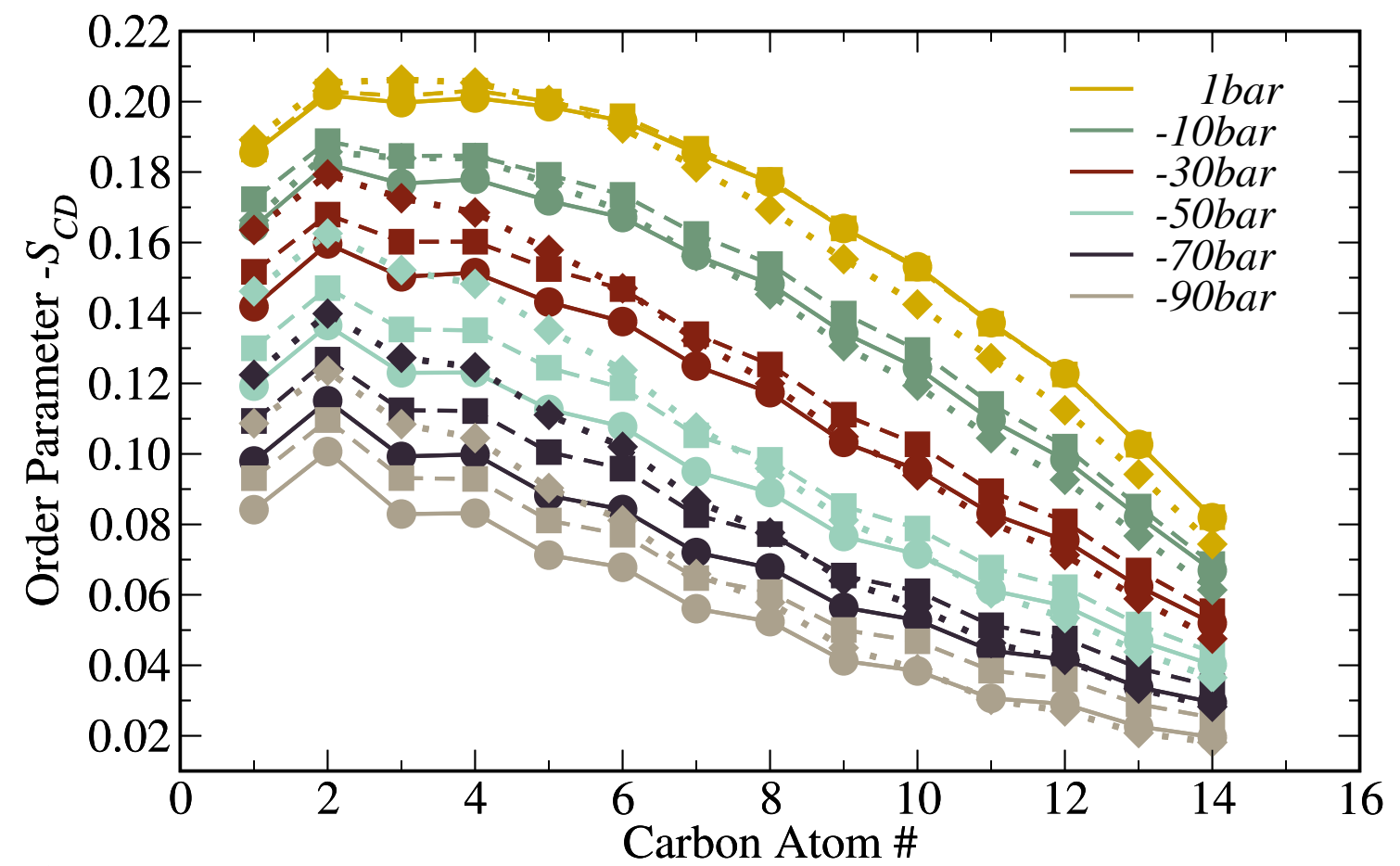

(b)

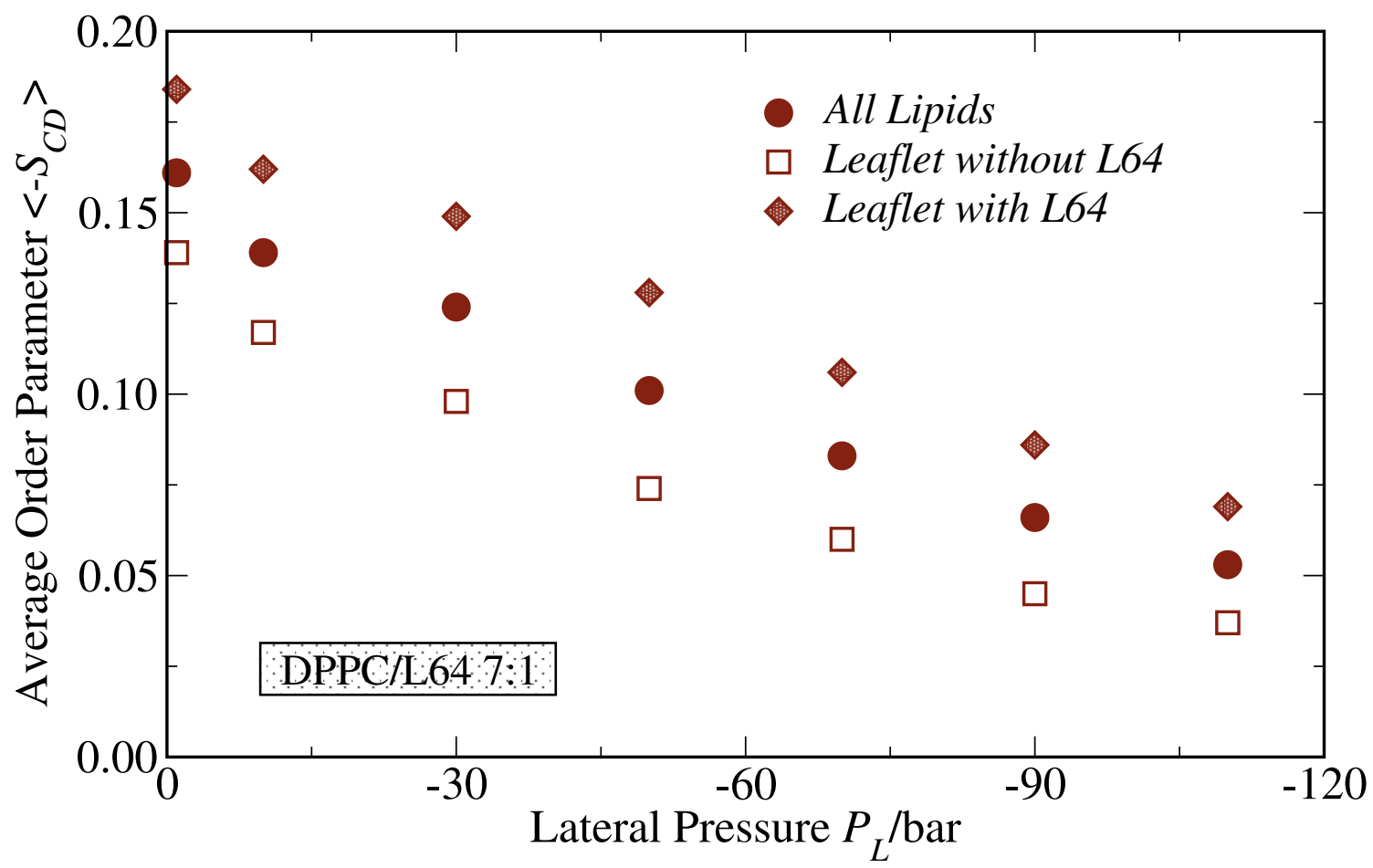

Figure 6: (a)Deuterium order parameter $\left(S_{C D}\right)$ profiles for the $s n-1$ lipid acyl tail. The carbon atom number increases towards the center of the bilayer. Solid line, filled circles polymer-free DPPC; dashed line, filled squares DPPC/L64 82:1; pointed line, filled diamonds DPPC/L64 7:1. (b) The average deuterium order parameter $\left(S_{C D}\right)$ for the HighHPL system, calculated for all the lipids (circles), for the leaflet without polymers (squares) and for the leaflet with polymers (diamonds). The calculations are averaged over the final $100 \mathrm{~ns}$ of the trajectory. 
(Figure S2). As the packing of the lipid tails is directly related to the total membrane area, the evident increase of the order parameter at high pressures and high lipid/polymer ratio could be linked to the lower areal strain that was observed for this system. Figure 6b reports the average deuterium order parameters $\left(\left\langle-S_{C D}\right\rangle\right)$ (averaged over all the acyl carbon atoms) for the HighHPL as a function of the applied pressure and calculated separately for the polymer-rich and the polymer-free leaflet. It can be clearly seen that the polymers force the lipids to remain ordered and (as we will see later) prevent their lateral diffusion. The difference in lipid packing properties between the polymer-rich and the polymer-free leaflet, has implications on the corresponding leaflet thickness. The thickness of each leaflet is calculated as the distance between the maximum and the minimum of the DPPC density profiles (Figure S3) and is shown in Figure 7. Increasing external lateral pressure induces a significant thinning on the polymer-free leaflet, whereas the polymer-rich leaflet maintains an almost constant thickness irrespectively of the area expansion.

\section{Effect of the L64 chains on the fluidity of the membrane}

A major implication of the inclusion of the polymer in the lipid bilayer is the alteration of the membrane fluidity. The membrane fluidity is related to the lipid lateral diffusion (i.e., in the membrane plane) $\left(D_{\text {lip }}\right)$ which can be quantified by the mean square displacement (MSD) of the lipid phosphate groups averaged over all the lipids and the final $200 \mathrm{~ns}$ of each trajectory (Figure $\mathrm{S} 4)$. The $\left(D_{\text {lip }}\right)$ can be calculated by employing the Einstein's relation:

$$
D_{l i p}=\lim _{t \rightarrow \infty} \frac{1}{2 D t}\left\langle\|\vec{r}(t)-\vec{r}(0)\|^{2}\right\rangle
$$

where $D$ equals 2 , as the calculation is restricted in two dimensions and $\vec{r}(\mathrm{t})$ is the position of the phosphorus atom after a time interval $t$. The diffusion coefficient is calculated from the slope of a least squares straight line fitted to the MSD curve in the region 20-180 ns. The $D_{l i p}$ in the pure DPPC and tension-free system is $8.1 \pm 0.4 \cdot 10^{-12} \mathrm{~m}^{2} \cdot \mathrm{s}^{-1}$, values 


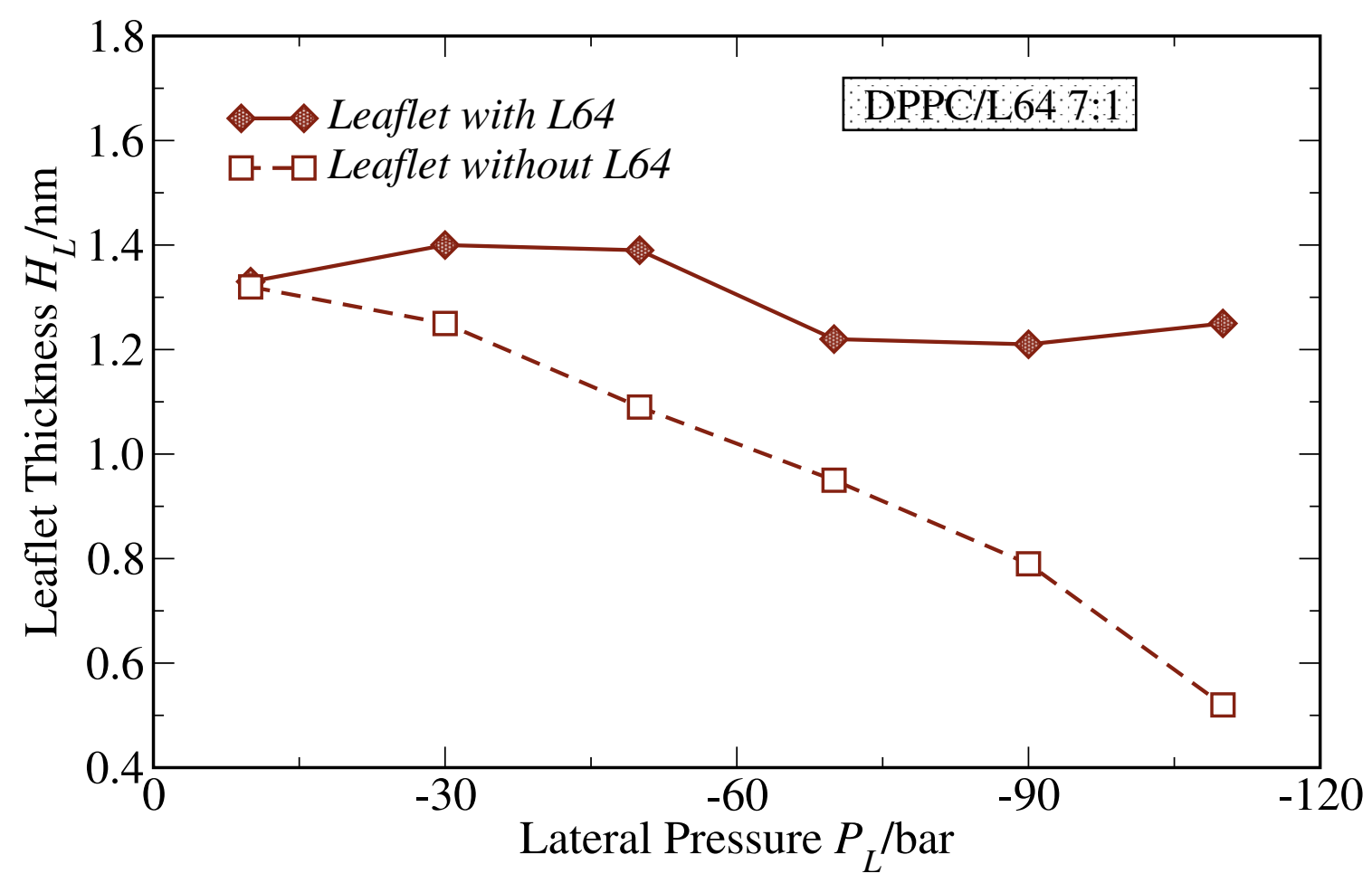

Figure 7: Membrane leaflet thickness $\left(H_{L}\right)$ versus the applied lateral pressures $\left(P_{L}\right)$, for the HighHPL system, calculated separately for the leaflet with (diamonds) and without (squares) polymers. The thickness was calculated as the distance between the maximum (headgroup region) and the minimum (bilayer center) in the phosphate density profiles. 
that compare well with the corresponding experimental data ${ }^{71}$ that lie within $1.5-6 \cdot 10^{-12} \mathrm{~m}^{2}$ $\cdot \mathrm{s}^{-1}$ and is in excellent agreement with previous computational values. ${ }^{44}$ The lipid lateral diffusivity increases when the bilayer is expanded as can be seen in Figure 8a. The increased free area around the lipid molecules facilitates their translational movement since, according to the free-area theory, ${ }^{72}$ the lipids can translate laterally when there is adjacent free space that exceeds a critical value. However, the mobility of the DPPC molecules when tension is applied increases less abruptly in the case of the hybrid membranes. Especially, for the high polymer concentration, the lipid displacement seems to be highly restricted and increases only moderately with the lateral pressure. A similar behaviour has been observed computationally ${ }^{67,68}$ for lipid membranes adsorbing relatively small molecules such as cholesterol or fullerenes and experimentally for supported membranes with adsorbed macromolecules. ${ }^{73,74}$ In particular, Granick and co-workers ${ }^{73,74}$ using spatially resolved measurements noticed a bimodal distribution of the lipid diffusion coefficient corresponding to slow and rapid diffusion. The slow movement was attributed to polymer-rich domains, where the lipids were bound to the polymer chains which dictate the lipid mobility. The small system size of the simulations does not allow the observation of only polymer-rich domains, but since the polymers are localized mainly in one of the leaflets, the diffusivity can be calculated separately for the polymer-rich and polymer-free monolayers in the high lipid/polymer ratio system. Interestingly, Figure 8b clearly shows that the lipids within the hybrid membrane diffuse with different rates; the polymer-bound lipids remain "trapped" by the chains and diffuse slowly, even for large area expansions, whereas the polymer-free lipids move faster with diffusion coefficients that approach those of the pure bilayer. The second interesting finding from Figure $8 \mathrm{~b}$ is that even when the bilayers are expanded, the lipid diffusion remains limited, despite the increase in the free area around them. 

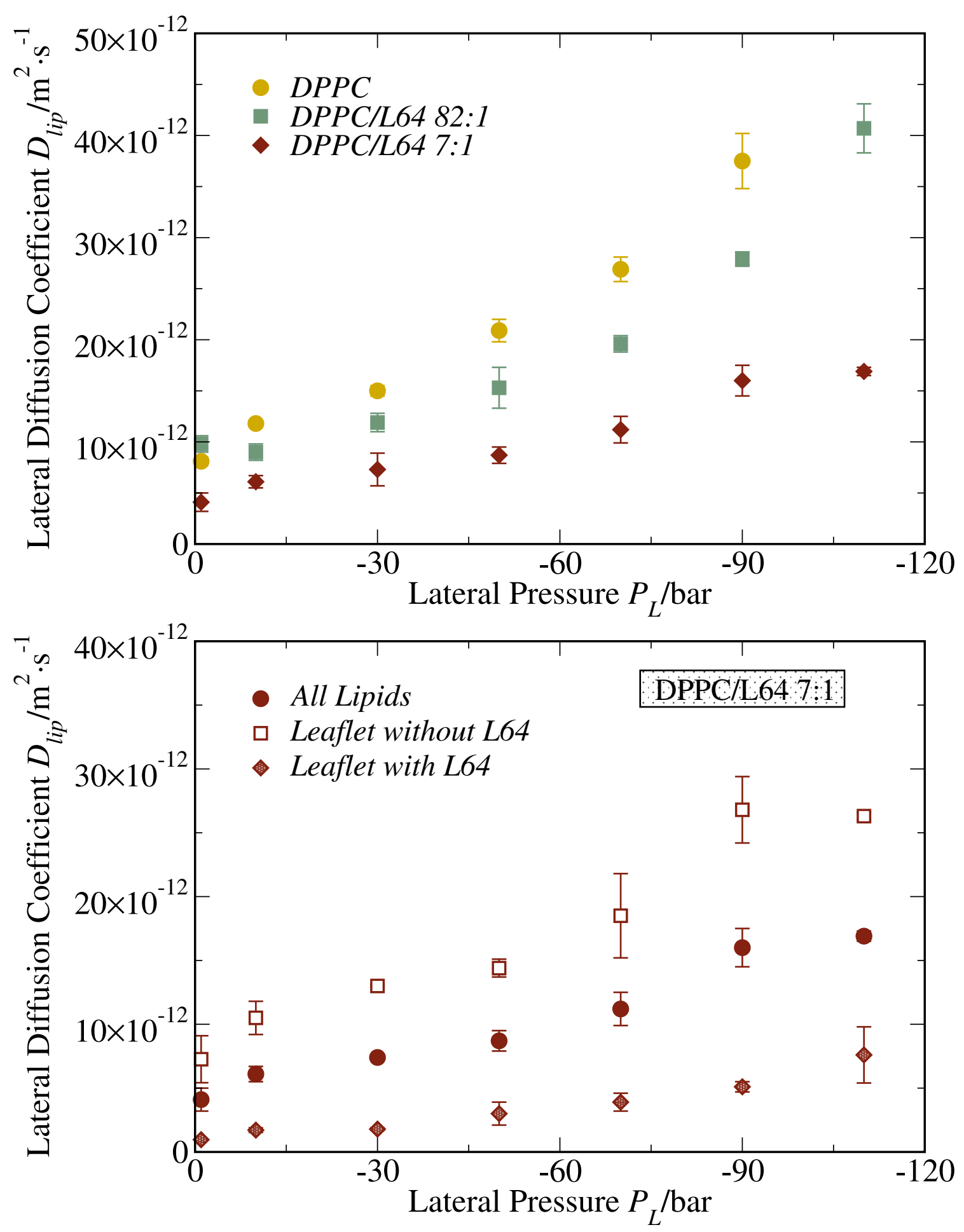

Figure 8: (a) Lipid lateral diffusion coefficients $\left(D_{\text {lip }}\right)$ versus the applied lateral pressures $\left(P_{L}\right)$, for all the DPPC/L64 ratios. (b) The lipid lateral diffusion coefficients $\left(D_{\text {lip }}\right)$ for the HighHPL system, calculated for all the lipids (circles), for the leaflet without polymers (squares) and for the leaflet with polymers (diamonds). 


\section{L64 chains induce lipid flip-flops}

The motion of lipids within bilayer structures is usually restricted in-plane for short time scales. The lipids can either move around their equilibrium position or diffuse laterally on the plane of the membrane when a void appears nearby due to thermal fluctuations. ${ }^{44} \mathrm{~A}$ more rare event however, is the transverse movement of a lipid that manages to overcome the energy barrier imposed to the headgroup by the hydrophobic tail region and diffuse to the opposite leaflet. This type of movement is known as flip-flop and cannot be typically observed within the short time frame of atomistic simulations. However, experimental studies have revealed the ability of Pluronic ${ }^{\circledR}$ polymers, to disturb the internal structure of bilayers and facilitate this translational movement of lipids. ${ }^{26}$ Our simulations reveal that the incorporation of Pluronic ${ }^{\circledR}$ chains within the membrane can indeed facilitate and accelerate lipid flip-flop. In the high polymer content system, at $P_{L}=-10$ bar, we observed two lipids from the polymer-rich bilayer to flip-flop to the polymer-free leaflet. Careful visualization of the trajectory revealed that the lipids are initially dragged to the tails region by a neighboring PEO block that also translocates deeper in the membrane.

The whole process begins after $150 \mathrm{~ns}$ and lasts until the end of the simulation at 500 ns (Figure 9) with the lipids occupying the space between the two leaflets most of the time and having lost their orientation along to the bilayer normal. The local bilayer structure is temporarily perturbed during the flip-flop movement of the lipids, but no water channel is generated. Flip-flops are also observed for the systems under high $P_{L}$, right before pore formation, when already the lipids have lost their ordering and the free volume is increased allowing them to move more freely. The flip-flop as investigated through experiments has shown to be correlated to the chemical structure of the incorporated polymers, with more hydrophobic Pluronic ${ }^{\circledR}$ having a significantly stronger effect. ${ }^{26}$ The simulations though show that the flip-flop is feasible when the PEO enters the membrane hydrophobic region and pulls the lipids in its vicinity by interacting favourably with their headgroups, suggesting that the polar block of the copolymers determines the flip-flop process. 


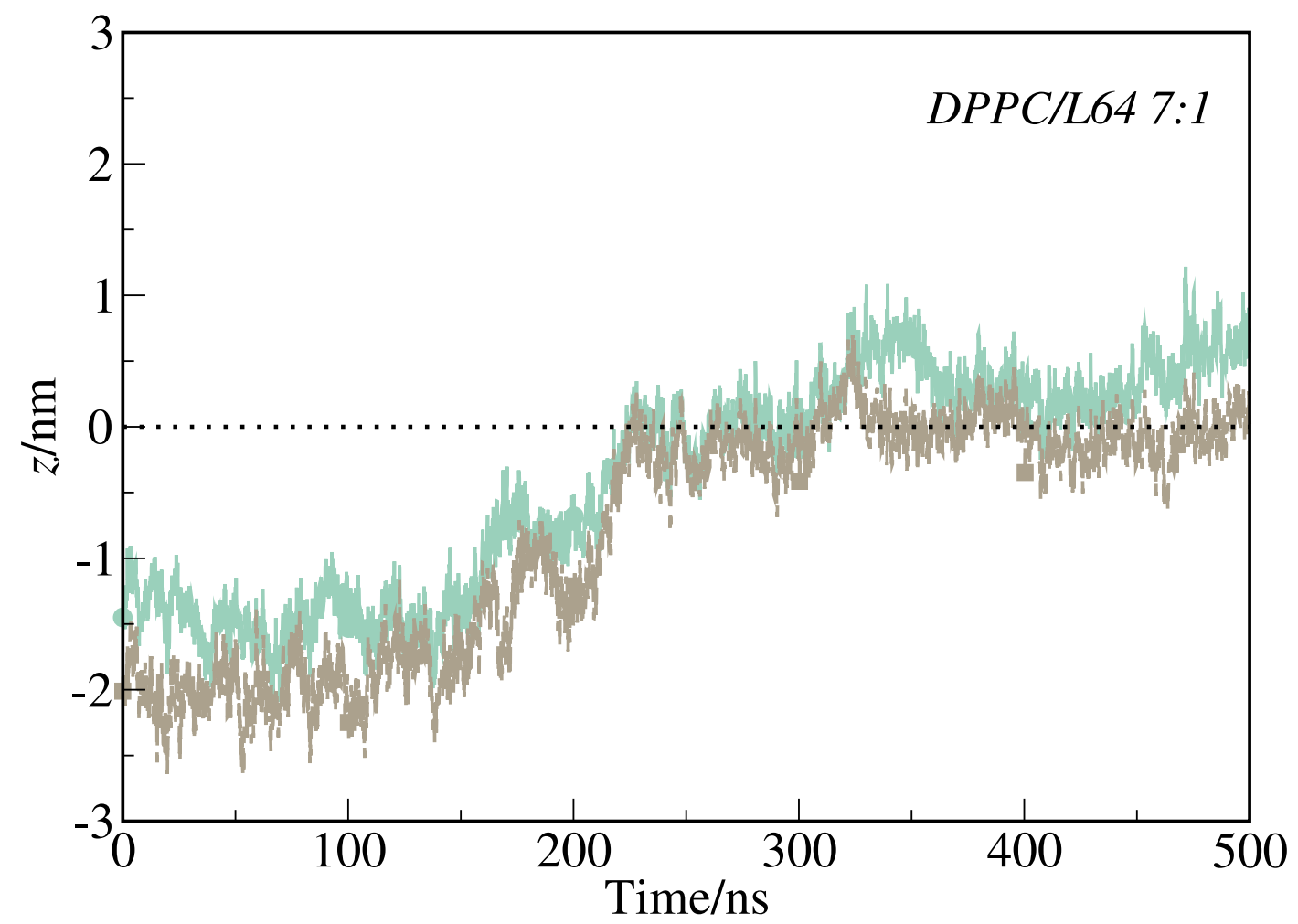

Figure 9: Time evolution of the distance on the $z$-axis of two lipids that flip-flop from the center of mass of the bilayer at $z=0$. 


\section{Pore Formation and Bilayer Rupture}

When the applied pressure exceeds a threshold value, the bilayer surface area becomes too large and the membranes lose their cohesive structure and break. The critical values of the rupture tensions and the rupture areal strain for the studied systems were determined by the tension-areal strain curves (Figure 3a). The DPPC bilayer breaks when $\gamma$ becomes 71.8 $\mathrm{mN} / \mathrm{m}$ and the $A_{\text {lip }}$ reaches $\sim 1.1 \mathrm{~nm}^{2}$, which corresponds to $\epsilon_{A}=0.73$. The critical area per lipid is comparable to the results of Leontiadou et al., ${ }^{63}$ who find the $A_{\text {lip }}$ of the rupture equal to $1.2 \mathrm{~nm}^{2}$. For the polymer-containing membranes, the critical $\gamma$ increases to 73.4 $\mathrm{mN} / \mathrm{m}$ and $75.8 \mathrm{mN} / \mathrm{m}$ for the LowHPL and the HighHPL, respectively, and the critical $\epsilon_{A}$ is between $0.70-0.77$ without showing a trend relative to the polymer concentration.

To gain an insight into the pore formation mechanism and test the reproducibility of the stages that lead to the membrane rupture, the simulations at the $P_{L c}$ were repeated three times and the same observations were made. In all systems, prior to rupture, a pore is formed and water penetrates from both leaflets creating a channel. Immediately after the first water pore is formed, more pores appear in the bilayer and they expand rapidly leading to complete distortion of the membrane structure. Even though the stress-strain curves seem to indicate that the polymer content does not affect significantly the critical rupture point (i.e., $\gamma$ is 2.4 $\mathrm{mN} / \mathrm{m}$ higher for the HighHPL than for the LowHPL), a more careful look into the trajectories reveals two entirely different lysis mechanisms. For the LowHPL membrane, the pore appears in a region far from the location where the polymer chain is adsorbed (Figure 10a) and is caused by the increased free volume that enhances water permeability and allows the water molecules to enter the hydrophobic region of the bilayer from both leaflets, disrupting the membrane structure. The same mechanism is observed in the polymer-free membrane but in the case of the LowHPL membrane, the polymer chain plays the role of reducing the local lipid diffusivity and thus the local area fluctuations hindering the pore formation in its vicinity. For the HighHPL membrane, the pore is located close to the polymer and induced by it. At first, the PEO block of one of the chains manages to overcome the energy barrier 
(a)

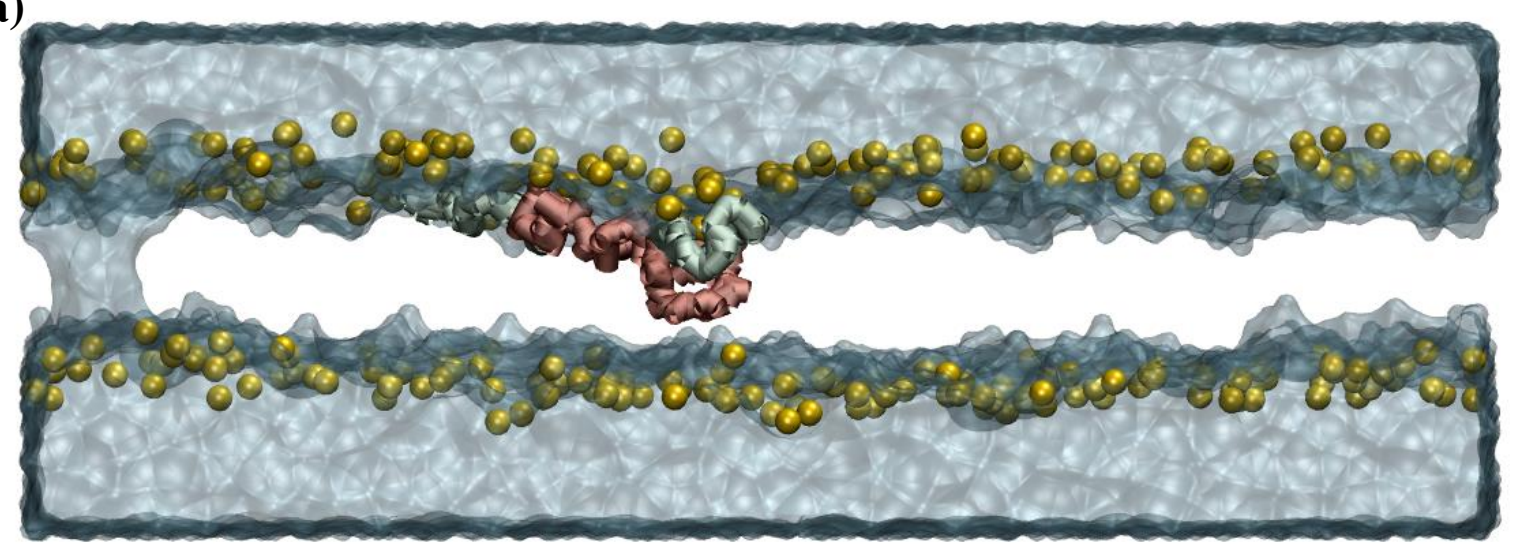

(b)

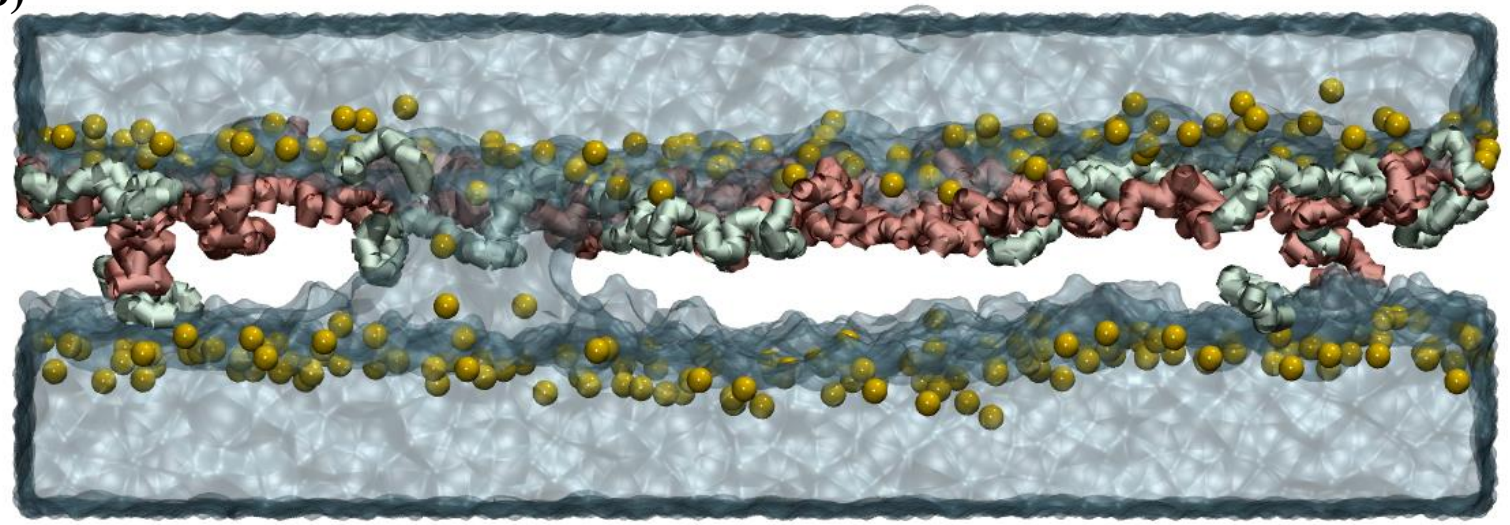

Figure 10: Side-view of the membranes after the water channels have been formed. (a) LowHPL bilayer and (b) HighHPL bilayer. For the color code refer to Fig. 2. For clarity, only the Phosphorus atoms of the lipids are shown. 
and moves towards the bilayer center. This PEO translocation seems to perturb the local lipid bilayer conformation, as a few nearby lipids lose their orientation and move towards the opposite leaflet. During the PEO rearrangement and the lipid flip-flop, water is dragged to the hydrophobic region of the membrane resulting in the formation of a water channel (Figure 10b). The pore again expands quickly and the bilayer breaks, similarly to the other systems.

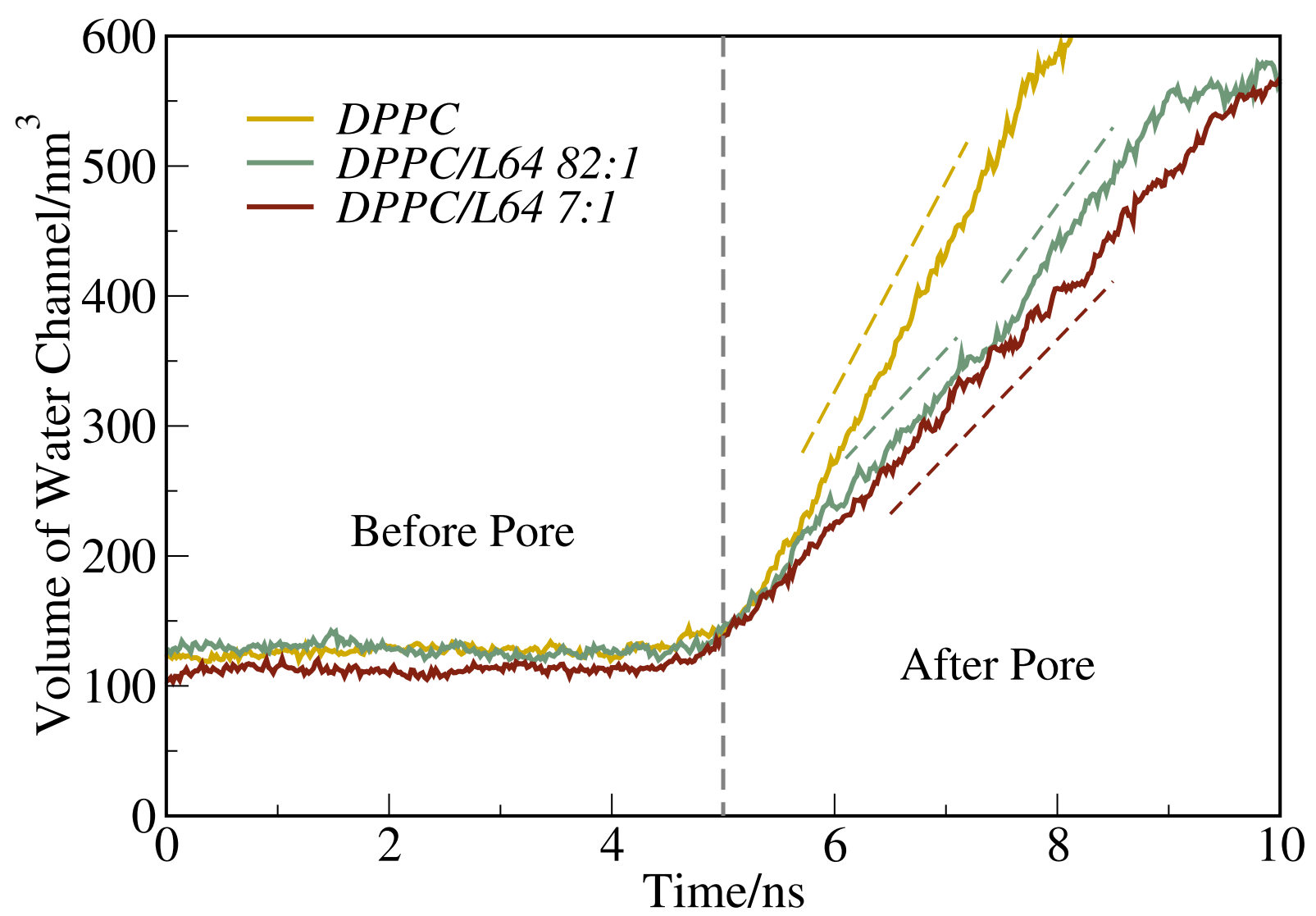

Figure 11: Water-occupied volume of the pores formed $\left(V_{\text {water }}\right)$ as a function of time, for 5 ns before and after the pore formation. The water-occupied volume prior to pore formation corresponds to the water molecules in the headgroup region.

The dynamics of the pore growth are also affected by the polymer chains. The volume of water in the bilayer region $\left(V_{\text {water }}\right)$ as a function of time is illustrated in Figure 11. The $V_{\text {water }}$ was calculated by counting the number of water molecules in the region between the phosphate groups of the two leaflets and multiplying by the volume of each molecule, calcu- 
lated considering the water atoms as spheres with radii equal to the Van der Waals radii. It can be seen that the rate of increase of the $V_{\text {water }}$ differs for the three investigated systems. Immediately after the appearance of the first pore, the growth of the water channel follows a linear trend with the slope corresponding to the rate of growth $\left(d V_{\text {water }} / d t\right)$. In the lipid membrane, the $V_{\text {water }}$ increases by $161 \mathrm{~nm}^{3} / \mathrm{ns}$, whereas for the HighHPL system, it is reduced to $89.5 \mathrm{~nm}^{3} / \mathrm{ns}$. In the LowHPL bilayer, initially the rate is calculated at $93.2 \mathrm{~nm}^{3} / \mathrm{ns}$, but less than 3 ns after the first pore, a second one is created, increasing the $d V_{\text {water }} / d t$ to $119.6 \mathrm{~nm}^{3} / \mathrm{ns}$. The slower diffusion of the lipids that are bound to the polymer chains is most likely the cause of the obstruction to the pore growth in the hybrid membranes and it reveals a potential capability of Pluronic ${ }^{\circledR}$ copolymers in stabilizing defects of lipid membranes, which will be the subject of a future study focused on the pore kinetics.

\section{Conclusions}

Our simulations have revealed that the incorporation of Pluronic ${ }^{\circledR}$ L64 chains in preformed lipid membranes can alter the membrane elastic properties under mechanical stress. The hybrid membranes resist the expansion of their surface area and the critical rupture tension increases from $71.8 \mathrm{mN} / \mathrm{m}$ (lipid bilayer) to $75.8 \mathrm{mN} / \mathrm{m}$ (HighHPL bilayer). The presence of the polymer chains hinder the lipids diffusion in their proximity and as a result, the lipids remain closely packed, even when the applied stress forces the membrane to expand and to increase its surface area. In the HPL membranes, the lipid tails show higher orientational order than the lipids in standard lipid membranes. This higher order of the fatty acid tails limits the thinning effect of the polymer-rich leaflets under stress, whereas the polymer-free leaflets are characterized by a decreased order and thickness. The increase in the compressibility modulus of the hybrid membranes suggests that they become stiffer and therefore mechanically stronger. The reduced lipid mobility and the increased stiffness are both pa- 
rameters that imply reduced permeability of small molecules, ${ }^{68}$ which is a desired property for the design of nanocarriers with low drug leakage during blood circulation. The presence of the L64 chains is also found to facilitate the trans-bilayer lipid motions (flip-flop), even at low lateral pressures, an event that is normally very rare (one flip-flop $/ 24 \mathrm{~h}^{75}$ ), verifying previous experimental observation. ${ }^{26}$ Regarding the formation of water pores, two different mechanisms were identified for the two polymer concentrations, that were driven by the distributions of the chains within the bilayer. The rate of growth of these pores is found to be reduced by the presence of polymer chains.

Our results suggest that the incorporation of amphiphilic block copolymers such as Pluronic ${ }^{\circledR}$ increases the mechanical stability of lipid membranes even at small concentration and indicate that the passive adsorption of polymers in preformed liposomes might be enough to construct hybrid vesicles with improved mechanical properties. Although our simulations have been performed on a specific Pluronic ${ }^{\circledR}$ copolymer, the details of the molecular mechanism driving the change in the mechanical and structural properties of the membrane are valid for any other copolymer of the Pluronic ${ }^{\circledR}$ family and in general for any copolymer containing PEO blocks that can translocate within the membrane.

\section{Acknowledgement}

The authors thank the N8 HPC Centre of Excellence, coordinated by the Universities of Leeds and Manchester for the computing time. This work has been sponsored by the Leverhulme grant number RPG-2013-246.

\section{Supporting Information Available}

The following files are available free of charge.

- supportinginfo.pdf: Supplementary figures. 
This material is available free of charge via the Internet at http://pubs.acs.org/.

\section{References}

(1) Lai, K.; Wang, B.; Zhang, Y.; Zheng, Y. Computer simulation study of nanoparticle interaction with a lipid membrane under mechanical stress. Physical Chemistry Chemical Physics 2013, 15, 270-278.

(2) Immordino, M. L.; Dosio, F.; Cattel, L. Stealth liposomes: review of the basic science, rationale, and clinical applications, existing and potential. International journal of nanomedicine 2006, 1, 297-315.

(3) Xiong, R.; Raemdonck, K.; Peynshaert, K.; Lentacker, I.; De Cock, I.; Demeester, J.; De Smedt, S. C.; Skirtach, A. G.; Braeckmans, K. Comparison of gold nanoparticle mediated photoporation: vapor nanobubbles outperform direct heating for delivering macromolecules in live cells. ACS nano 2014, 8, 6288-6296.

(4) Choudhury, C. K.; Kumar, A.; Roy, S. Characterization of conformation and interaction of gene delivery vector polyethylenimine with phospholipid bilayer at different protonation state. Biomacromolecules 2013, 14, 3759-3768.

(5) Hadinoto, K.; Sundaresan, A.; Cheow, W. S. Lipid-polymer hybrid nanoparticles as a new generation therapeutic delivery platform: a review. European journal of pharmaceutics and biopharmaceutics 2013, 85, 427-443.

(6) Krishnamurthy, S.; Vaiyapuri, R.; Zhang, L.; Chan, J. M. Lipid-coated polymeric nanoparticles for cancer drug delivery. Biomaterials science 2015, 3, 923-936.

(7) Daoulas, K. C.; Müller, M. Polymer membranes/biomembranes; Springer, 2009; pp $43-85$. 
(8) Shen, W.; Hu, J.; Hu, X. Impact of amphiphilic triblock copolymers on stability and permeability of phospholipid/polymer hybrid vesicles. Chemical Physics Letters 2014, $600,56-61$.

(9) Quemeneur, F.; Rinaudo, M.; Pépin-Donat, B. Influence of polyelectrolyte chemical structure on their interaction with lipid membrane of zwitterionic liposomes. Biomacromolecules 2008, 9, 2237-2243.

(10) Quemeneur, F.; Rinaudo, M.; Maret, G.; Pépin-Donat, B. Decoration of lipid vesicles by polyelectrolytes: mechanism and structure. Soft Matter 2010, 6, 4471-4481.

(11) Mandal, B.; Bhattacharjee, H.; Mittal, N.; Sah, H.; Balabathula, P.; Thoma, L. A.; Wood, G. C. Core-shell-type lipid-polymer hybrid nanoparticles as a drug delivery platform. Nanomedicine: Nanotechnology, Biology and Medicine 2013, 9, 474-491.

(12) Chen, D.; Santore, M. M. Hybrid copolymer-phospholipid vesicles: phase separation resembling mixed phospholipid lamellae, but with mechanical stability and control. Soft Matter 2015, 11, 2617-2626.

(13) Sullan, R. M. A.; Shi, W.; Chan, H.; Li, J. K.; Walker, G. C. Mechanical stability of phase-segregated multicomponent lipid bilayers enhanced by PS-b-PEO diblock copolymers. Soft Matter 2013, 9, 6245-6253.

(14) Zhang, R. X.; Cai, P.; Zhang, T.; Chen, K.; Li, J.; Cheng, J.; Pang, K. S.; Adissu, H. A.; Rauth, A. M.; Wu, X. Y. Polymer-lipid hybrid nanoparticles synchronize pharmacokinetics of co-encapsulated doxorubicin-mitomycin $\mathrm{C}$ and enable their spatiotemporal co-delivery and local bioavailability in breast tumor. Nanomedicine: Nanotechnology, Biology and Medicine 2016, 12, 1279-1290.

(15) Pitto-Barry, A.; Barry, N. P. Pluronic® block-copolymers in medicine: from chemical and biological versatility to rationalisation and clinical advances. Polymer Chemistry 2014, 5, 3291-3297. 
(16) Tomasini, M. D.; Rinaldi, C.; Tomassone, M. S. Molecular dynamics simulations of rupture in lipid bilayers. Experimental Biology and Medicine 2010, 235, 181-188.

(17) Nawaz, S.; Redhead, M.; Mantovani, G.; Alexander, C.; Bosquillon, C.; Carbone, P. Interactions of PEO-PPO-PEO block copolymers with lipid membranes: a computational and experimental study linking membrane lysis with polymer structure. Soft Matter 2012, 8, 6744-6754.

(18) Groot, R. D.; Rabone, K. Mesoscopic simulation of cell membrane damage, morphology change and rupture by nonionic surfactants. Biophysical journal 2001, 81, 725-736.

(19) Moldovan, D.; Pinisetty, D.; Devireddy, R. V. Molecular dynamics simulation of pore growth in lipid bilayer membranes in the presence of edge-active agents. Applied Physics Letters 2007, 91, 204104.

(20) Hezaveh, S.; Samanta, S.; De Nicola, A.; Milano, G.; Roccatano, D. Understanding the interaction of block copolymers with DMPC lipid bilayer using coarse-grained molecular dynamics simulations. The Journal of Physical Chemistry B 2012, 116, 14333-14345.

(21) Ileri Ercan, N.; Stroeve, P.; Tringe, J. W.; Faller, R. Understanding the Interaction of Pluronics L61 and L64 with a DOPC Lipid Bilayer: An Atomistic Molecular Dynamics Study. Langmuir 2016, 32, 10026-10033.

(22) Rabbel, H.; Werner, M.; Sommer, J.-U. Interactions of amphiphilic triblock copolymers with lipid membranes: modes of interaction and effect on permeability examined by generic Monte Carlo simulations. Macromolecules 2015, 48, 4724-4732.

(23) Srinivas*, G.; Klein, M. L. Coarse-grain molecular dynamics simulations of diblock copolymer surfactants interacting with a lipid bilayer. Molecular Physics 2004, 102, 883-889. 
(24) Tomasini, M. D.; Tomassone, M. S. Dissipative particle dynamics simulation of poly (ethylene oxide)-poly (ethyl ethylene) block copolymer properties for enhancement of cell membrane rupture under stress. Chemical engineering science 2012, 71, 400-408.

(25) Rossi, G.; Monticelli, L. Simulating the interaction of lipid membranes with polymer and ligand-coated nanoparticles. Advances in Physics: X 2016, 1, 276-296.

(26) Demina, T.; Grozdova, I.; Krylova, O.; Zhirnov, A.; Istratov, V.; Frey, H.; Kautz, H.; Melik-Nubarov, N. Relationship between the structure of amphiphilic copolymers and their ability to disturb lipid bilayers. Biochemistry 2005, 44, 4042-4054.

(27) Lee, B.; Firestone, M. A. Electron density mapping of triblock copolymers associated with model biomembranes: insights into conformational states and effect on bilayer structure. Biomacromolecules 2008, 9, 1541-1550.

(28) Redhead, M.; Mantovani, G.; Nawaz, S.; Carbone, P.; Gorecki, D. C.; Alexander, C.; Bosquillon, C. Relationship between the affinity of PEO-PPO-PEO block copolymers for biological membranes and their cellular effects. Pharmaceutical research 2012, 29, $1908-1918$.

(29) Wu, G.; Majewski, J.; Ege, C.; Kjaer, K.; Weygand, M. J.; Lee, K. Y. C. Lipid corralling and poloxamer squeeze-out in membranes. Physical review letters 2004, 93, 028101/1$028101 / 4$.

(30) Newman, M. J.; Balusubramanian, M.; Todd, C. W. Development of adjuvant-active nonionic block copolymers. Advanced drug delivery reviews 1998, 32, 199-223.

(31) Kositza, M. J.; Bohne, C.; Alexandridis, P.; Hatton, T. A.; Holzwarth, J. F. Micellization dynamics and impurity solubilization of the block-copolymer L64 in an aqueous solution. Langmuir 1999, 15, 322-325. 
(32) Kloxin, C. J.; van Zanten, J. H. Microviscoelasticity of adhesive hard sphere dispersions: Tracer particle microrheology of aqueous Pluronic L64 solutions. The Journal of chemical physics 2009, 131, 134904.

(33) Sezgin-Bayindir, Z.; Antep, M. N.; Yuksel, N. Development and characterization of mixed niosomes for oral delivery using candesartan cilexetil as a model poorly watersoluble drug. AAPS PharmSciTech 2015, 16, 108-117.

(34) Tavano, L.; Rossi, C. O.; Picci, N.; Muzzalupo, R. Spontaneous temperature-sensitive Pluronic $\AA$ based niosomes: Triggered drug release using mild hyperthermia. International journal of pharmaceutics 2016, 511, 703-708.

(35) Ghaouar, N.; Baroudi, M.; Othman, T. Contribution to the explanation of the association process of two triblock poly (ethylene oxide)-poly (propylene oxide)-poly (ethylene oxide)(PEO-PPO-PEO) copolymers and their mixtures in an aqueous solution. Journal of Molecular Liquids 2016, 224, 279-283.

(36) Shukr, M. H. Novel in situ gelling ocular inserts for voriconazole-loaded niosomes: design, in vitro characterisation and in vivo evaluation of the ocular irritation and drug pharmacokinetics. Journal of microencapsulation 2016, 33, 71-79.

(37) Gong, H.; Xu, L.; Zhu, T.; Xu, G.; Shi, X.; Dong, M.; Li, Y. Interactions between pluronic block polyether and CTAB at air/water interface: interfacial dilational rheology study. Colloid and Polymer Science 2016, 294, 1577-1584.

(38) Ramírez, P.; Stocco, A.; Muñoz, J.; Miller, R. Interfacial rheology and conformations of triblock copolymers adsorbed onto the water-oil interface. Journal of colloid and interface science 2012, 378, 135-143.

(39) Nagle, J. F.; Tristram-Nagle, S. Structure of lipid bilayers. Biochimica et Biophysica Acta (BBA)-Reviews on Biomembranes 2000, 1469, 159-195. 
(40) Venable, R. M.; Brown, F. L.; Pastor, R. W. Mechanical properties of lipid bilayers from molecular dynamics simulation. Chemistry and physics of lipids 2015, 192, 60-74.

(41) Lee, S.-M.; Ahn, R. W.; Chen, F.; Fought, A. J.; OâĂŹhalloran, T. V.; Cryns, V. L.; Nguyen, S. T. Biological evaluation of $\mathrm{pH}-$ responsive polymer-caged nanobins for breast cancer therapy. ACS nano 2010, 4, 4971-4978.

(42) Berger, O.; Edholm, O.; Jähnig, F. Molecular dynamics simulations of a fluid bilayer of dipalmitoylphosphatidylcholine at full hydration, constant pressure, and constant temperature. Biophysical journal 1997, 72, 2002-2013.

(43) Berendsen, H. J.; Postma, J. P.; van Gunsteren, W. F.; Hermans, J. Intermolecular forces; Springer, 1981; pp 331-342.

(44) Muddana, H. S.; Gullapalli, R. R.; Manias, E.; Butler, P. J. Atomistic simulation of lipid and DiI dynamics in membrane bilayers under tension. Physical Chemistry Chemical Physics 2011, 13, 1368-1378.

(45) Hoover, W. G. Canonical dynamics: equilibrium phase-space distributions. Physical review A 1985, 31, 1695-1697.

(46) Nosé, S. A molecular dynamics method for simulations in the canonical ensemble. Molecular physics 1984, 52, 255-268.

(47) Nosé, S. A unified formulation of the constant temperature molecular dynamics methods. The Journal of chemical physics 1984, 81, 511-519.

(48) Parrinello, M.; Rahman, A. Polymorphic transitions in single crystals: A new molecular dynamics method. Journal of Applied physics 1981, 52, 7182-7190.

(49) Nosé, S.; Klein, M. L. A study of solid and liquid carbon tetrafluoride using the constant pressure molecular dynamics technique. The Journal of Chemical Physics 1983, 78, 6928-6939. 
(50) Hess, B. P-LINCS: A parallel linear constraint solver for molecular simulation. Journal of Chemical Theory and Computation 2008, 4, 116-122.

(51) Darden, T.; York, D.; Pedersen, L. Particle mesh Ewald: An NâŃĚ log (N) method for Ewald sums in large systems. The Journal of chemical physics 1993, 98, 10089-10092.

(52) Hess, B.; Kutzner, C.; Van Der Spoel, D.; Lindahl, E. GROMACS 4: algorithms for highly efficient, load-balanced, and scalable molecular simulation. Journal of chemical theory and computation 2008, 4, 435-447.

(53) Humphrey, W.; Dalke, A.; Schulten, K. VMD: visual molecular dynamics. Journal of molecular graphics 1996, 14, 33-38.

(54) Michaud-Agrawal, N.; Denning, E. J.; Woolf, T. B.; Beckstein, O. MDAnalysis: a toolkit for the analysis of molecular dynamics simulations. Journal of computational chemistry 2011, 32, 2319-2327.

(55) Gowers, R. J.; Linke, M.; Barnoud, J.; Reddy, T. J.; Melo, M. N.; Seyler, S. L.; Dotson, D.; Domanski, J.; Buchoux, S.; Kenney, I. M. MDAnalysis: a Python package for the rapid analysis of molecular dynamics simulations. Proceedings of the 15th Python in Science Conference, Austin, TX. 2016.

(56) Torcello-Gómez, A.; Wulff-Pérez, M.; Gálvez-Ruiz, M. J.; Martín-Rodríguez, A.; Cabrerizo-Vílchez, M.; Maldonado-Valderrama, J. Block copolymers at interfaces: Interactions with physiological media. Advances in colloid and interface science $\mathbf{2 0 1 4}$, $206,414-427$.

(57) Torcello-GoİĄmez, A.; Maldonado-Valderrama, J.; JoİĄdar-Reyes, A. B.; Foster, T. J. Interactions between pluronics (F127 and F68) and bile salts (NaTDC) in the aqueous phase and the interface of oil-in-water emulsions. Langmuir 2013, 29, 2520-2529. 
(58) Ramírez, P.; Muñoz, J.; Fainerman, V.; Aksenenko, E.; Mucic, N.; Miller, R. Dynamic interfacial tension of triblock copolymers solutions at the water-hexane interface. Colloids and Surfaces A: Physicochemical and Engineering Aspects 2011, 391, 119-124.

(59) Taddese, T.; Carbone, P.; Cheung, D. L. Thermodynamics of linear and star polymers at fluid interfaces. Soft matter 2015, 11, 81-93.

(60) Taddese, T.; Carbone, P. Effect of Chain Length on the Partition Properties of Poly (ethylene oxide): Comparison between MARTINI Coarse-Grained and Atomistic Models. The Journal of Physical Chemistry B 2017, 121, 1601-1609.

(61) Needham, D.; Nunn, R. S. Elastic deformation and failure of lipid bilayer membranes containing cholesterol. Biophysical journal 1990, 58, 997-1009.

(62) Xie, J. Y.; Ding, G. H.; Karttunen, M. Molecular dynamics simulations of lipid membranes with lateral force: rupture and dynamic properties. Biochimica et Biophysica Acta (BBA)-Biomembranes 2014, 1838, 994-1002.

(63) Leontiadou, H.; Mark, A. E.; Marrink, S. J. Molecular dynamics simulations of hydrophilic pores in lipid bilayers. Biophysical journal 2004, 86, 2156-2164.

(64) Evans, E.; Heinrich, V. Dynamic strength of fluid membranes. Comptes Rendus Physique 2003, 4, 265-274.

(65) Barbe, S.; Lafaquiere, V.; Guieysse, D.; Monsan, P.; Remaud-Siméon, M.; Andre, I. Insights into lid movements of Burkholderia cepacia lipase inferred from molecular dynamics simulations. Proteins: Structure, Function, and Bioinformatics 2009, 77, $509-523$.

(66) Feller, S. E.; Pastor, R. W. Constant surface tension simulations of lipid bilayers: the sensitivity of surface areas and compressibilities. The Journal of chemical physics $\mathbf{1 9 9 9 ,}$ $111,1281-1287$. 
(67) Wong-Ekkabut, J.; Baoukina, S.; Triampo, W.; Tang, I.-M.; Tieleman, D. P.; Monticelli, L. Computer simulation study of fullerene translocation through lipid membranes. Nature Nanotechnology 2008, 3, 363-368.

(68) Falck, E.; Patra, M.; Karttunen, M.; Hyvönen, M. T.; Vattulainen, I. Lessons of slicing membranes: interplay of packing, free area, and lateral diffusion in phospholipid/cholesterol bilayers. Biophysical journal 2004, 87, 1076-1091.

(69) Flyvbjerg, H. Advances in Computer Simulation; Springer, 1998; pp 88-103.

(70) Vermeer, L. S.; De Groot, B. L.; Réat, V.; Milon, A.; Czaplicki, J. Acyl chain order parameter profiles in phospholipid bilayers: computation from molecular dynamics simulations and comparison with 2H NMR experiments. European Biophysics Journal 2007, 36, 919-931.

(71) Gullapalli, R. R.; Demirel, M. C.; Butler, P. J. Molecular dynamics simulations of DiI-C 18 (3) in a DPPC lipid bilayer. Physical Chemistry Chemical Physics 2008, 10, 3548-3560.

(72) Galla, H.-J.; Hartmann, W.; Theilen, U.; Sackmann, E. On two-dimensional passive random walk in lipid bilayers and fluid pathways in biomembranes. The Journal of membrane biology 1979, 48, 215-236.

(73) Zhang, L.; Granick, S. Slaved diffusion in phospholipid bilayers. Proceedings of the National Academy of Sciences of the United States of America 2005, 102, 9118-9121.

(74) Zhang, L.; Granick, S. Dynamical heterogeneity in supported lipid bilayers. MRS bulletin 2006, 31, 527-531.

(75) Contreras, F.; Sánchez-Magraner, L.; Alonso, A.; Goñi, F. M. Transbilayer (flip-flop) lipid motion and lipid scrambling in membranes. FEBS letters 2010, 584, 1779-1786. 


\section{Graphical TOC Entry}

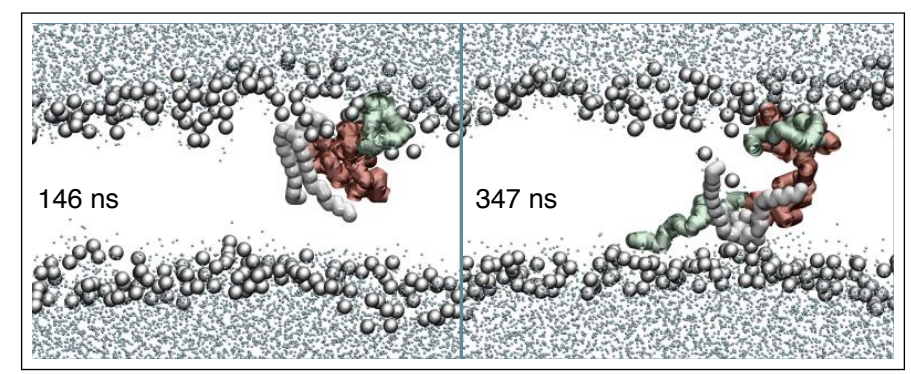

20

21 